\title{
Two-step hyperentanglement purification with the quantum-state-joining method*
}

\author{
Bao-Cang Ren, Fang-Fang Du, and Fu-Guo Deng ${ }^{\dagger}$ \\ Department of Physics, Applied Optics Beijing Area Major Laboratory, \\ Beijing Normal University, Beijing 100875, China
}

(Dated: September 25, 2018)

\begin{abstract}
Hyperentanglement is a promising resource in quantum information processing, especially for increasing the channel capacity of long-distance quantum communication. Hyperentanglement purification is an important method to obtain high-fidelity nonlocal hyperentangled states from mixed hyperentangled states in a long-distance quantum communication process with noisy channels. Here, we present a two-step hyperentanglement purification protocol for nonlocal mixed hyperentangled states with polarization bit-flip errors and spatial-mode phase-flip errors, resorting to polarization-spatial phase-check quantum nondemolition detectors and the quantum-state-joining method (QSJM). With QSJM, the protocol can preserve the states that are discarded in the previous hyperentanglement purification protocols. It has the advantage of a high efficiency, and it is useful for improving the entanglement of photon systems with several degrees of freedom in longdistance high-capacity quantum communication.

PACS numbers: 03.67.Pp, 03.67.Bg, 03.65.Yz, 03.67.Hk
\end{abstract}

\section{INTRODUCTION}

Entanglement is an essential quantum resource in quantum information processing, and it improves the methods of manipulating and transmitting information in quantum computation and quantum communication [1]. The maximally entangled photon system, used as a quantum channel, is a core resource in quantum communication [2 13]. With maximally entangled photon pairs, the two remote parties in quantum communication, say Alice and Bob, can implement faithful teleportation [2]. That is, they teleport the unknown quantum state of a single particle without moving the particle itself [2]. Also, Alice can transmit two or more bits of information to Bob by moving only a photon back and forth with quantum dense coding protocols based on a maximally entangled photon pair [3, 4]. They can create a private key with maximally entangled photon pairs by resorting to quantum key distribution protocols [5 7], even in the case with a collective-noise optical-fiber channel [8]. With quantum secure direct communication protocols based on entangled photon pairs [9, 10], Alice and Bob can also transmit their secret message directly, without creating a private key in advance. With entanglement-based quantum secret-sharing protocols [1-13], the parties in quantum communication can share some private keys with a potentially dishonest party.

Hyperentanglement, defined as the entanglement in several degrees of freedom (DOFs) of a quantum system [14 16], has attracted much attention for improving the power of quantum computation (e.g., hyperparallel photonic quantum computation [17, 18]) and increasing the channel capacity of long-distance quan-

\footnotetext{
*Published in Phys. Rev. A 90, 052309 (2014)

${ }^{\dagger}$ Corresponding author: fgdeng@bnu.edu.cn
}

tum communication, such as photonic superdense coding with polarization-orbital-angular-momentum hyperentanglement [19], quantum teleportation with photon pairs entangled in two DOFs [20], entanglement swapping based on photonic spatial-polarization hyperentanglement [20, 21], hyperentangled Bell-state analysis [20 24], hyperentanglement concentration 25, 26], and so on. With two-photon hyperentanglement, the GreenbergerHorne-Zeilinger (GHZ) theorem has been generalized to the case with only two entangled particles [27], and the first two-particle all-or-nothing test of local realism has been demonstrated experimentally with a linear optics setup, without resorting to a noncontextuality assumption [28]. Hyperentanglement has also been used to assist long-distance quantum communication in one DOF of photon systems, such as a quantum repeater 29], complete Bell-state analysis 30 34], and deterministic entanglement purification [34 38] on the polarizations of photon pairs. In long-distance quantum communication protocols, the photon signals can be transmitted no more than several hundreds of kilometers over an optical fiber or a free space, and quantum repeaters are required in this condition. The (hyper)entangled photon systems are always produced locally, and they inevitably suffer from environment noise in their distribution process and storage process in quantum communication. In general, the maximally (hyper)entangled photon states may decay into less entangled pure states or even into mixed states, which decreases the fidelity and the security of long-distance quantum communication protocols.

Entanglement purification is an interesting passive way to suppress the effect of environmental noise in quantum communication processes. It is used to distill some quantum systems in high-fidelity entangled states from those in less-entangled mixed states [34 54]. In 1996, Bennett et al. [39] introduced the first entanglement purification protocol (EPP) for quantum systems in a Werner state with quantum controlled-not (CNOT) gates. In 2001, 
Pan et al. [49] presented a polarization EPP with linear optics. In 2002, Simon and Pan proposed a polarization EPP assisted by hyperentanglement with the available parametric down-conversion (PDC) resource [50], which was demonstrated by Pan et al. [51] in 2003. In 2008, Sheng et al. [41] proposed an efficient polarization EPP with hyperentanglement based on a PDC resource, resorting to cross-Kerr nonlinearity. In 2010, Sheng and Deng 34] introduced the concept of deterministic entanglement purification for two-photon systems with hyperentanglement. Subsequently, some interesting deterministic EPPs were proposed [35 38] with linear optical elements. The EPPs for multipartite entangledphoton systems in mixed-polarization GHZ states have also been investigated with nonlinear optical elements in the past few years [42, 43]. In 2013, a hyperentanglement purification protocol (hyper-EPP) was proposed for two-photon systems in mixed polarization-spatial hyperentangled Bell states with polarization bit-flip errors and spatial-mode bit-flip errors resorting to nonlinear optical elements [46]. However, it is implemented by purifying the polarization states and the spatial-mode states of photon pairs independently. In 2013, Vitelli et al. 55] demonstrated experimentally the quantum-state-joining process with linear optical elements, which can combine the two-dimensional quantum states of two input photons into an output single photon.

In this paper, we present a two-step high-efficiency hyper-EPP for nonlocal photon systems in mixed polarization-spatial hyperentangled states, resorting to the polarization-spatial phase-check quantum nondemolition detectors (P-S-QNDs) and the quantum-statejoining method (QSJM). Our P-S-QND is implemented with the hybrid CNOT gate [18] based on the giant optical circular birefringence (GOCB) of the quantum-dot (QD) spin inside a double-sided optical microcavity (a double-sided QD-cavity system). Our QSJM is also constructed with the GOCB of a double-sided QD-cavity system. With our QSJM, mixed hyperentangled states with only one DOF in the preserving condition can be combined into mixed hyperentangled states with two DOFs in the preserving condition, and the efficiency of our twostep hyper-EPP can be greatly improved by preserving the states that are discarded in the previous hyper-EPP without QSJM. QSJM is very useful for improving the entanglement of photon systems in long-distance quantum communication.

This paper is organized as follows: In Sec. IIA, we introduce the GOCB of a quantum-dot spin inside a double-sided optical microcavity. Based on GOCB, we give the details of our QSJM and our P-S-QND in Secs. IIB and IIC respectively. In Sec. III, we introduce our two-step high-efficiency hyper-EPP for nonlocal twophoton systems with QSJM. Its generalization for hyperentangled three-photon GHZ states is given in Sec. IV] A discussion and a summary are given in Sec. V In Appendixes $\mathrm{A}$ and $\mathrm{B}$, we give the QSJM for hyperentangled Bell states and a detailed calculation for the fidelities and the efficiencies of P-S-QND and QSJM, respectively.

\section{QUANTUM-STATE-JOINING METHOD AND POLARIZATION-SPATIAL PHASE-CHECK QND}

\author{
A. Giant optical circular birefringence of a \\ quantum-dot electron spin in a double-sided optical \\ resonant microcavity
}

The QSJM and the P-S-QND in our proposal are both constructed with the optical property of a singly charged QD [e.g., a self-assembled $\operatorname{In}(\mathrm{Ga}) \mathrm{As}$ QD or GaAs interfacial QD] embedded in a double-sided optical resonant microcavity, as shown in Fig. 1(a). The two distributed Bragg reflectors of the double-sided optical resonant microcavity are partially reflective and low loss for on-resonance transmission, which supports both polarization modes of photon systems [56]. If an excess electron is injected into the QD, the singly charged QD has optical resonance with the creation of a negatively charged exciton $X^{-}$, which consists of two antiparallel electron spins bound to one hole [57], and it shows spindependent transitions with circularly polarized lights according to Pauli's exclusion principle [58] [shown in Fig. 1(b)]. If the excess electron is in the spin state $|\uparrow\rangle$, a circularly polarized photon with spin $S_{z}=+1$ is absorbed to create the negatively charged exciton $X^{-}$in the state $|\uparrow \downarrow \uparrow\rangle$. However, for the excess electron-spin state $|\downarrow\rangle$, a circularly polarized photon with $\operatorname{spin} S_{z}=-1$ is absorbed to create the negatively charged exciton $X^{-}$in the state $|\downarrow \uparrow \Downarrow\rangle$. Here $|\Uparrow\rangle(|\Downarrow\rangle)$ represents the heavyhole spin $\left|+\frac{3}{2}\right\rangle\left(\left|-\frac{3}{2}\right\rangle\right)$, and $|\uparrow\rangle(|\downarrow\rangle)$ represents the excess electron spin $\left|+\frac{1}{2}\right\rangle\left(\left|-\frac{1}{2}\right\rangle\right)$.

The input-output optical property of the double-sided QD-cavity system can be described by the Heisenberg equations of motion for the cavity field operator $\hat{a}$ and the $X^{-}$dipole operator $\hat{\sigma}_{-}$in the interaction picture [59, 60],

$$
\begin{aligned}
\frac{d \hat{a}}{d t}= & -\left[i\left(\omega_{c}-\omega\right)+\kappa+\frac{\kappa_{s}}{2}\right] \hat{a}-g \hat{\sigma}_{-} \\
& -\sqrt{\kappa} \hat{a}_{i n}-\sqrt{\kappa} \hat{a}_{i n}^{\prime}, \\
\frac{d \hat{\sigma}_{-}}{d t}= & -\left[i\left(\omega_{X^{-}}-\omega\right)+\frac{\gamma}{2}\right] \hat{\sigma}_{-}-g \hat{\sigma}_{z} \hat{a}, \\
\hat{a}_{r}= & \hat{a}_{i n}+\sqrt{\kappa} \hat{a}, \\
\hat{a}_{t}= & \hat{a}_{i n}^{\prime}+\sqrt{\kappa} \hat{a},
\end{aligned}
$$

where $\omega_{c}, \omega$, and $\omega_{X^{-}}$are the frequencies of the cavity mode, the input photon, and the $X^{-}$transition, respectively. $g$ is the coupling strength of the negatively charged exciton $X^{-}$and the cavity mode. $\gamma / 2$ is the decay rate of $X^{-} . \kappa$ is the decay rate of the cavity field mode, and $\kappa_{s} / 2$ is the decay rate of the cavity field mode to the cavity side leakage mode. $\hat{a}_{i n}, \hat{a}_{i n}^{\prime}$ and $\hat{a}_{r}, \hat{a}_{t}$ are the input and output field operators of the double-sided QD-cavity system, respectively. 


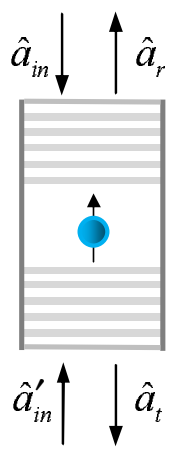

(a)

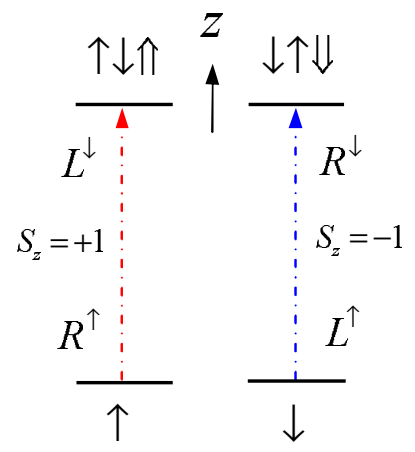

(b)
FIG. 1: (Color online) The optical transition of a QD embedded in a double-sided microcavity with circularly polarized photons. (a) A double-sided QD-cavity system. The QD is located at the center of a double-sided cavity for maximal lightmatter coupling. (b) The spin-dependent optical transitions of a negatively charged exciton $X^{-}$with circularly polarized photons. $L^{\uparrow}\left(L^{\downarrow}\right)$ and $R^{\uparrow}\left(R^{\downarrow}\right)$ represent the left- and rightcircularly polarized lights with their input directions parallel (antiparallel) to the $\mathrm{z}$ direction, respectively. $\uparrow \downarrow \uparrow(\downarrow \uparrow \downarrow)$ represents the spin state $\left|+\frac{3}{2}\right\rangle\left(\left|-\frac{3}{2}\right\rangle\right)$ of the negatively charged exciton $X^{-} \cdot \uparrow(\downarrow)$ represents the spin state $\left|+\frac{1}{2}\right\rangle\left(\left|-\frac{1}{2}\right\rangle\right)$ of the excess electron in a QD.

In the weak excitation condition, $X^{-}$is dominantly in the ground state with $\left\langle\sigma_{z}\right\rangle=-1$, and the reflection $[r(\omega)]$ and transmission $[t(\omega)]$ coefficients of the doublesided QD-cavity system are described by [60]

$$
\begin{aligned}
r(\omega) & =1+t(\omega), \\
t(\omega) & =\frac{-\kappa\left[i\left(\omega_{X^{-}}-\omega\right)+\frac{\gamma}{2}\right]}{\left[i\left(\omega_{X^{-}}-\omega\right)+\frac{\gamma}{2}\right]\left[i\left(\omega_{c}-\omega\right)+\kappa+\frac{\kappa_{s}}{2}\right]+g^{2}} .
\end{aligned}
$$

In the resonant condition $\left(\omega_{c}=\omega_{X^{-}}=\omega_{0}\right)$, if the coupling strength is $g=0$ (the QD is decoupled from the cavity), the reflection and transmission coefficients can be expressed as [60]

$$
\begin{aligned}
& r_{0}(\omega)=\frac{i\left(\omega_{0}-\omega\right)+\frac{\kappa_{s}}{2}}{i\left(\omega_{0}-\omega\right)+\kappa+\frac{\kappa_{s}}{2}}, \\
& t_{0}(\omega)=\frac{-\kappa}{i\left(\omega_{0}-\omega\right)+\kappa+\frac{\kappa_{s}}{2}} .
\end{aligned}
$$

In the strong-coupling regime $[g>(\kappa, \gamma)]$ and the resonant condition $\left(\omega_{c}=\omega_{X^{-}}=\omega_{0} \approx \omega\right)$, if the cavity side leakage is neglected, the reflection and transmission coefficients can achieve $|r| \rightarrow 1,\left|r_{0}\right| \rightarrow 0$ and $|t| \rightarrow 0$, $\left|t_{0}\right| \rightarrow 1$.

As the photonic circular polarization usually depends on the direction of propagation, the photon in state $\left|R^{\uparrow}\right\rangle$ or $\left|L^{\downarrow}\right\rangle$ has spin $S_{z}=+1$, and the photon in state $\left|R^{\downarrow}\right\rangle$ or $\left|L^{\uparrow}\right\rangle$ has spin $S_{z}=-1$. Here $L^{\uparrow}\left(L^{\downarrow}\right)$ and $R^{\uparrow}\left(R^{\downarrow}\right)$ represent the left- and right-circularly polarized lights with their input directions parallel (antiparallel) to the $\mathrm{z}$ direction, respectively, shown in Fig. 1(b). For the electron spin state $|\uparrow\rangle$, the input circularly polarized photon $\left|R^{\uparrow}\right\rangle$ $\left(\left|L^{\downarrow}\right\rangle\right)$ is reflected as $\left|L^{\downarrow}\right\rangle\left(\left|R^{\uparrow}\right\rangle\right)$ by the QD-cavity system with the QD coupled to the cavity, while the input circularly polarized photon $\left|R^{\downarrow}\right\rangle\left(\left|L^{\uparrow}\right\rangle\right)$ is transmitted through the QD-cavity system with a phase shift relative to the reflected photon (QD is decoupled from the cavity). For the electron spin state $|\downarrow\rangle$, the input circularly polarized photon $\left|R^{\uparrow}\right\rangle\left(\left|L^{\downarrow}\right\rangle\right)$ is transmitted through the QD-cavity system, and the input circularly polarized photon $\left|R^{\downarrow}\right\rangle$ $\left(\left|L^{\uparrow}\right\rangle\right)$ is reflected by the QD-cavity system. That is, the reflection and transmission rules of the photon polarization states can be summarized as follows [18, 60]:

$$
\begin{array}{ll}
\left|R^{\uparrow}, i_{2}, \uparrow\right\rangle \rightarrow\left|L^{\downarrow}, i_{2}, \uparrow\right\rangle, & \left|L^{\downarrow}, i_{1}, \uparrow\right\rangle \rightarrow\left|R^{\uparrow}, i_{1}, \uparrow\right\rangle, \\
\left|R^{\uparrow}, i_{2}, \downarrow\right\rangle \rightarrow-\left|R^{\uparrow}, i_{1} \downarrow\right\rangle, & \left|L^{\downarrow}, i_{1}, \downarrow\right\rangle \rightarrow-\left|L^{\downarrow}, i_{2}, \downarrow\right\rangle, \\
\left|R^{\downarrow}, i_{1}, \uparrow\right\rangle \rightarrow-\left|R^{\downarrow}, i_{2}, \uparrow\right\rangle, & \left|L^{\uparrow}, i_{2}, \uparrow\right\rangle \rightarrow-\left|L^{\uparrow}, i_{1}, \uparrow\right\rangle, \\
\left|R^{\downarrow}, i_{1}, \downarrow\right\rangle \rightarrow\left|L^{\uparrow}, i_{1}, \downarrow\right\rangle, & \left|L^{\uparrow}, i_{2}, \downarrow\right\rangle \rightarrow\left|R^{\downarrow}, i_{2}, \downarrow\right\rangle .
\end{array}
$$

Here $i_{1}$ and $i_{2}(i=a, b)$ are the two spatial modes of photon $i$ (shown in Fig. 2).

\section{B. Quantum-state-joining method}

The QSJM for photonic states introduced here is used to transfer the polarization state of photon $A$ to the polarization state of photon $B$ without disturbing the spatial-mode state of photon $B$, resorting to the reflection-transmission optical property of the doublesided QD-cavity system, as shown in Fig. 2(a). The initial states of the two photons, $A$ and $B$, are

$$
\begin{aligned}
& \left|\phi_{A}\right\rangle=\left(\alpha_{1}|R\rangle+\alpha_{2}|L\rangle\right)_{A}\left(\gamma_{1}\left|a_{1}\right\rangle+\gamma_{2}\left|a_{2}\right\rangle\right), \\
& \left|\phi_{B}\right\rangle=\left(\beta_{1}|R\rangle+\beta_{2}|L\rangle\right)_{B}\left(\delta_{1}\left|b_{1}\right\rangle+\delta_{2}\left|b_{2}\right\rangle\right) .
\end{aligned}
$$

The excess electron spin in the QD is prepared in the state $|+\rangle_{e}=\frac{1}{\sqrt{2}}(|\uparrow\rangle+|\downarrow\rangle)_{e}$.

We put photon $A$ into the circularly polarizing beam splitter $\mathrm{CPBS}_{1}$, wave plate $\mathrm{U}_{1}, \mathrm{QD}, \mathrm{U}_{2}, \mathrm{CPBS}_{2}$, and half-wave plate $\mathrm{X}$ in sequence, as shown in Fig. 2(a). After photon $A$ passes through the quantum circuit shown in Fig. 2(a), the state of the quantum system composed of a $\mathrm{QD}$ and photon $A$ is transformed from $\left|\phi_{A e}\right\rangle_{0}$ to $\left|\phi_{A e}\right\rangle_{1}$. Here

$$
\begin{aligned}
\left|\phi_{A e}\right\rangle_{0}= & |+\rangle_{e} \otimes\left|\phi_{A}\right\rangle, \\
\left|\phi_{A e}\right\rangle_{1}= & \frac{1}{\sqrt{2}}\left[|R\rangle_{A}\left(\alpha_{1}|\uparrow\rangle+\alpha_{2}|\downarrow\rangle\right)_{e}+|L\rangle_{A}\left(\alpha_{2}|\uparrow\rangle\right.\right. \\
& \left.\left.+\alpha_{1}|\downarrow\rangle\right)_{e}\right]\left(\gamma_{1}\left|a_{1}\right\rangle+\gamma_{2}\left|a_{2}\right\rangle\right) .
\end{aligned}
$$

By measuring the polarization state of photon $A$ in the orthogonal basis $\{|R\rangle,|L\rangle\}$, the polarization state of photon $A$ is transferred to the excess electron spin state in the QD.

We assume the excess electron spin in the QD is in the state $|\phi\rangle_{e}=\left(\alpha_{1}|\uparrow\rangle+\alpha_{2}|\downarrow\rangle\right)_{e}$ (i.e., the polarization state of photon $A$ is projected into state $|R\rangle)$. Photon $B$ is put into the quantum circuit shown in Fig. 2(a) 


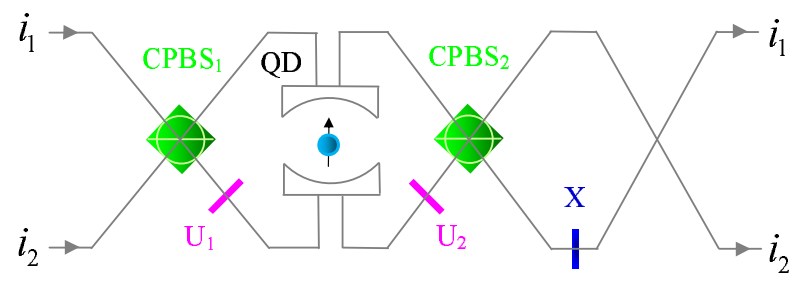

(a)

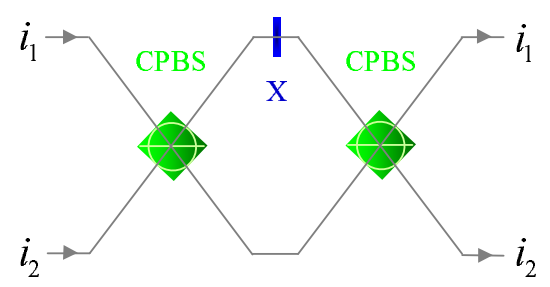

(b)

FIG. 2: (Color online) (a) Schematic diagram of our quantumstate-joining method (QSJM). (b) Schematic diagram of the swap gate between the spatial-mode state and the polarization state of a photon. QD represents a double-sided QD-cavity system. $\mathrm{CPBS}_{i}(i=1,2)$ represents a polarizing beam splitter in the circular basis, which transmits the right-circular polarization photon $|R\rangle$ and reflects the left-circular polarization photon $|L\rangle . \quad \mathrm{U}_{i}(i=1,2)$ represents a wave plate which is used to perform a polarization phase-flip operation $U=-|R\rangle\langle R|-| L\rangle\langle L|$ on a photon. X represents a half-wave plate which is used to perform a polarization bit-flip operation $\sigma_{x}^{P}=|R\rangle\langle L|+| L\rangle\langle R|$ on the photon passing through it. $i_{1}$ and $i_{2}$ represent the two spatial modes of photon $i(i=a, b)$.

after a Hadamard operation $\left[|\uparrow\rangle \rightarrow \frac{1}{\sqrt{2}}(|\uparrow\rangle+|\downarrow\rangle)\right.$, $\left.|\downarrow\rangle \rightarrow \frac{1}{\sqrt{2}}(|\uparrow\rangle-|\downarrow\rangle)\right]$ is performed on the excess electron spin in the QD, and the state of the quantum system composed of the $\mathrm{QD}$ and photon $B$ is transformed from $\left|\phi_{B e}\right\rangle_{1}$ to $\left|\phi_{B e}\right\rangle_{2}$. Here

$$
\begin{aligned}
\left|\phi_{B e}\right\rangle_{1}= & \left|\phi_{B}\right\rangle \otimes\left|\phi_{e}\right\rangle, \\
\left|\phi_{B e}\right\rangle_{2}= & {\left[\alpha_{1}^{\prime}|\uparrow\rangle_{e}\left(\beta_{1}|R\rangle+\beta_{2}|L\rangle\right)_{B}+\alpha_{2}^{\prime}|\downarrow\rangle_{e}\right.} \\
& \left.\left(\beta_{2}|R\rangle+\beta_{1}|L\rangle\right)_{B}\right]\left(\delta_{1}\left|b_{1}\right\rangle+\delta_{2}\left|b_{2}\right\rangle\right),
\end{aligned}
$$

where $\alpha_{1}^{\prime}=\frac{1}{\sqrt{2}}\left(\alpha_{1}+\alpha_{2}\right)$ and $\alpha_{2}^{\prime}=\frac{1}{\sqrt{2}}\left(\alpha_{1}-\alpha_{2}\right)$. After the Hadamard operations are performed on the polarization DOF of photon $B$ and the excess electron spin $e$ in sequence, we have photon $B$ pass through the quantum circuit shown in Fig.2(a) again, and the state of the quantum system $B e$ is changed to $\left|\phi_{B e}\right\rangle_{3}$. Here

$$
\begin{aligned}
\left|\phi_{B e}\right\rangle_{3}= & {\left[\alpha_{1}|R\rangle_{B}\left(\beta_{1}^{\prime}|\uparrow\rangle+\beta_{2}^{\prime}|\downarrow\rangle\right)_{e}+\alpha_{2}|L\rangle_{B}\right.} \\
& \left.\left(\beta_{2}^{\prime}|\uparrow\rangle+\beta_{1}^{\prime}|\downarrow\rangle\right)_{e}\right]\left(\delta_{1}\left|b_{1}\right\rangle+\delta_{2}\left|b_{2}\right\rangle\right),
\end{aligned}
$$

where $\beta_{1}^{\prime}=\frac{1}{\sqrt{2}}\left(\beta_{1}+\beta_{2}\right)$ and $\beta_{2}^{\prime}=\frac{1}{\sqrt{2}}\left(\beta_{1}-\beta_{2}\right)$. Finally, a Hadamard operation is performed on the excess electron spin $e$, and the state of the quantum system $B e$ is changed from $\left|\phi_{B e}\right\rangle_{3}$ to be $\left|\phi_{B e}\right\rangle_{4}$. Here

$$
\left|\phi_{B e}\right\rangle_{4}=\left[\beta_{1}|\uparrow\rangle_{e}\left(\alpha_{1}|R\rangle+\alpha_{2}|L\rangle\right)_{B}+\beta_{2}|\downarrow\rangle_{e}\right.
$$

$$
\left.\left(\alpha_{1}|R\rangle-\alpha_{2}|L\rangle\right)_{B}\right]\left(\delta_{1}\left|b_{1}\right\rangle+\delta_{2}\left|b_{2}\right\rangle\right) .
$$

By measuring the excess electron spin $e$ in the orthogonal basis $\{|\uparrow\rangle,|\downarrow\rangle\}$, the state of the excess electron spin $e\left[|\phi\rangle_{e}=\left(\alpha_{1}|\uparrow\rangle+\alpha_{2}|\downarrow\rangle\right)_{e}\right]$ is transferred to the polarization state of photon $B$ without disturbing its spatial-mode state. If the polarization DOF of photon $A$ is projected into state $|L\rangle$, a bit-flip operation $\left(\sigma_{x}^{P}=|R\rangle\langle L|+| L\rangle\langle R|\right)$ is performed on the polarization DOF of photon $B$, and a phase-flip operation $\left(\sigma_{z}^{P}=\right.$ $|R\rangle\langle R|-| L\rangle\langle L|)$ is performed on the polarization DOF of photon $B$ if the excess electron spin $e$ is projected into state $|\downarrow\rangle_{e}$. After the conditional operations are performed on the polarization DOF of photon $B$, the state of photon $B$ is changed to $\left|\phi_{B}\right\rangle_{f}=\left(\alpha_{1}|R\rangle+\alpha_{2}|L\rangle\right)_{B}\left(\delta_{1}\left|b_{1}\right\rangle+\delta_{2}\left|b_{2}\right\rangle\right)$. This is the result of the QSJM for combining the polarization state of photon $A$ and the spatial-mode state of photon $B$ into an output single photon.

The QSJM can also be used to transfer the spatialmode state of photon $A$ to the polarization state of photon $B$. This requires that photon $A$ passes through the quantum circuit shown in Fig. 2(b), which can swap the polarization state and the spatial-mode state of photon $A$. Subsequently, we can transfer the polarization state of photon $A$ to the polarization state of photon $B$ with the quantum circuit shown in Fig. 2(a). Then the result of the QSJM for combining the spatial-mode state of photon $A$ and the spatial-mode state of photon $B$ into a single output photon is achieved.

\section{Polarization-spatial phase-check QND}

Now, we introduce the construction of the polarizationspatial phase-check QND, which is used to distinguish the hyperentangled Bell states with the relative phase 0 from those with the relative phase $\pi$ in both the polarization and the spatial-mode DOFs. Our P-S-QND is implemented with the hybrid CNOT gate introduced in our previous work [18], and its setup is shown in Fig. 3(a). The 16 polarization-spatial hyperentangled Bell states are defined as $\left|\phi_{k l}\right\rangle_{A B}=\left|\phi_{k}\right\rangle_{A B}^{P} \otimes\left|\phi_{l}\right\rangle_{A B}^{S}(k, l=1,2,3,4)$, where

$$
\begin{aligned}
\left|\phi_{1}\right\rangle_{A B}^{P} & =\frac{1}{\sqrt{2}}(|R R\rangle+|L L\rangle)_{A B}, \\
\left|\phi_{2}\right\rangle_{A B}^{P} & =\frac{1}{\sqrt{2}}(|R R\rangle-|L L\rangle)_{A B}, \\
\left|\phi_{3}\right\rangle_{A B}^{P} & =\frac{1}{\sqrt{2}}(|R L\rangle+|L R\rangle)_{A B}, \\
\left|\phi_{4}\right\rangle_{A B}^{P} & =\frac{1}{\sqrt{2}}(|R L\rangle-|L R\rangle)_{A B}, \\
\left|\phi_{1}\right\rangle_{A B}^{S} & =\frac{1}{\sqrt{2}}\left(\left|a_{1} b_{1}\right\rangle+\left|a_{2} b_{2}\right\rangle\right), \\
\left|\phi_{2}\right\rangle_{A B}^{S} & =\frac{1}{\sqrt{2}}\left(\left|a_{1} b_{1}\right\rangle-\left|a_{2} b_{2}\right\rangle\right), \\
\left|\phi_{3}\right\rangle_{A B}^{S} & =\frac{1}{\sqrt{2}}\left(\left|a_{1} b_{2}\right\rangle+\left|a_{2} b_{1}\right\rangle\right),
\end{aligned}
$$




$$
\left|\phi_{4}\right\rangle_{A B}^{S}=\frac{1}{\sqrt{2}}\left(\left|a_{1} b_{2}\right\rangle-\left|a_{2} b_{1}\right\rangle\right) .
$$

Here the subscript $A B$ represents the photon pair $A B$, and the superscripts $P$ and $S$ represent the polarization and the spatial-mode DOFs of a photon system, respectively.

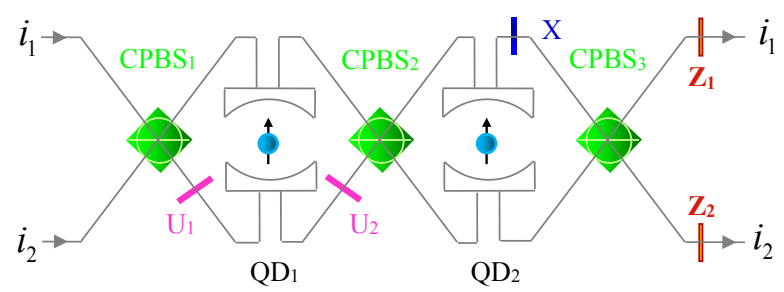

(a)

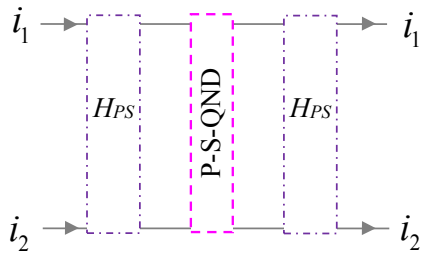

(b)

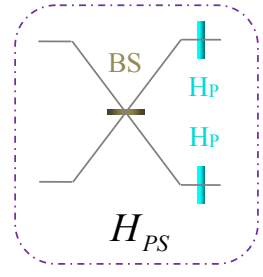

(c)
FIG. 3: (Color online) (a) Schematic diagram of the polarization-spatial phase-check QND (P-S-QND). (b) Schematic diagram of the polarization-spatial parity-check QND. (c) Schematic diagram of $H_{P S}$, which is used to perform the Hadamard operation on both the polarization and the spatial-mode DOFs of a photon. BS represents a 50:50 beam splitter which is used to perform the Hadamard operation $\left[\left|i_{1}\right\rangle \rightarrow \frac{1}{\sqrt{2}}\left(\left|i_{1}\right\rangle+\left|i_{2}\right\rangle\right),\left|i_{2}\right\rangle \rightarrow \frac{1}{\sqrt{2}}\left(\left|i_{1}\right\rangle-\left|i_{2}\right\rangle\right)\right]$ on the spatial-mode DOF of a photon. $\mathrm{Z}_{i}(i=1,2)$ represents a halfwave plate which is used to perform the polarization phase-flip operation $\sigma_{z}^{P}=-|R\rangle\langle R|+| L\rangle\langle L|$ on a photon. $H_{P}$ represents a half-wave plate which is used to perform the Hadamard operation $\left[|R\rangle \rightarrow \frac{1}{\sqrt{2}}(|R\rangle+|L\rangle),|L\rangle \rightarrow \frac{1}{\sqrt{2}}(|R\rangle-|L\rangle)\right]$ on the polarization DOF of a photon.

The initial states of the excess electron spins $e_{1}$ in $\mathrm{QD}_{1}$ and $e_{2}$ in $\mathrm{QD}_{2}$ are $|+\rangle_{e_{1}}=\frac{1}{\sqrt{2}}(|\uparrow\rangle+|\downarrow\rangle)_{e_{1}}$ and $|+\rangle_{e_{2}}=$ $\frac{1}{\sqrt{2}}(|\uparrow\rangle+|\downarrow\rangle)_{e_{2}}$, respectively. If we have photons $A$ and $B$ pass through the quantum circuit of the hybrid CNOT gate shown in Fig. 3(a) in sequence, the state of the quantum system composed of photon pair $A B$ and the excess electron spins $e_{1}$ and $e_{2}$ (in $\mathrm{QD}_{1}$ and $\mathrm{QD}_{2}$, respectively) is transformed into

$$
\begin{aligned}
\left|\phi_{k_{1} l_{1}}\right\rangle_{A B}|+\rangle_{e_{1}}|+\rangle_{e_{2}} & \rightarrow\left|\phi_{k_{1} l_{1}}\right\rangle_{A B}|+\rangle_{e_{1}}|-\rangle_{e_{2}}, \\
\left|\phi_{k_{1} l_{2}}\right\rangle_{A B}|+\rangle_{e_{1}}|+\rangle_{e_{2}} & \rightarrow\left|\phi_{k_{1} l_{2}}\right\rangle_{A B}|+\rangle_{e_{1}}|+\rangle_{e_{2}}, \\
\left|\phi_{k_{2} l_{1}}\right\rangle_{A B}|+\rangle_{e_{1}}|+\rangle_{e_{2}} & \rightarrow\left|\phi_{k_{2} l_{1}}\right\rangle_{A B}|-\rangle_{e_{1}}|-\rangle_{e_{2}}, \\
\left|\phi_{k_{2} l_{2}}\right\rangle_{A B}|+\rangle_{e_{1}}|+\rangle_{e_{2}} & \rightarrow\left|\phi_{k_{2} l_{2}}\right\rangle_{A B}|-\rangle_{e_{1}}|+\rangle_{e_{2}},
\end{aligned}
$$

where $k_{1}\left(l_{1}\right)=1,3, k_{2}\left(l_{2}\right)=2,4$, and $| \pm\rangle=\frac{1}{\sqrt{2}}(|\uparrow\rangle \pm|\downarrow\rangle)$. By measuring the spin states of excess electrons $e_{1}$ and $e_{2}$ in the orthogonal basis $\{|+\rangle,|-\rangle\}$ [18], one can distinguish the hyperentangled Bell states with the relative phase 0 from those with the relative phase $\pi$ in both the polarization and the spatial-mode DOFs. If the excess electron spin $e_{1}\left(e_{2}\right)$ is projected into state $|+\rangle_{e_{1}}\left(|-\rangle_{e_{2}}\right)$, the polarization (spatial-mode) state of the hyperentangled Bell state has the relative phase 0. Otherwise the polarization (spatial-mode) state of the hyperentangled Bell state has the relative phase $\pi$.

If we perform Hadamard operations on both the spatial-mode and the polarization DOFs of photons $A$ and $B$ before (and after) we put them into the quantum circuit of our P-S-QND shown in Fig. 3(b), the oddparity mode (i.e., $\left|\phi_{3}\right\rangle_{A B}^{P},\left|\phi_{4}\right\rangle_{A B}^{P},\left|\phi_{3}\right\rangle_{A B}^{S}$, and $\left|\phi_{4}\right\rangle_{A B}^{S}$ ) and the even-parity mode (i.e., $\left|\phi_{1}\right\rangle_{A B}^{P},\left|\phi_{2}\right\rangle_{A B}^{P},\left|\phi_{1}\right\rangle_{A B}^{S}$, and $\left.\left|\phi_{2}\right\rangle_{A B}^{S}\right)$ of the hyperentangled Bell states can be distinguished in both the polarization and the spatial-mode DOFs, which is the result of the polarization-spatial parity-check QND.

\section{TWO-STEP HYPER-EPP FOR MIXED HYPERENTANGLED BELL STATES WITH THE QUANTUM-STATE-JOINING METHOD}

In this section, we introduce our two-step hyperentanglement purification for mixed hyperentangled Bell states with polarization bit-flip errors and spatial-mode phaseflip errors, resorting to the P-S-QND and QSJM introduced in Sec. III. The setup of our two-step hyper-EPP with QSJM is shown in Fig. 4. It includes two steps in each round of the hyper-EPP process, and they are discussed in detail as follows.

\section{A. The first step of our two-step hyper-EPP with P-S-QNDs}

In this step, two identical two-photon systems in a mixed hyperentangled Bell state are required. They are described as follows:

$$
\begin{aligned}
\rho_{A B}= & {\left[F_{1}\left|\phi_{1}\right\rangle_{A B}^{P}\left\langle\phi_{1}\left|+\left(1-F_{1}\right)\right| \phi_{3}\right\rangle_{A B}^{P}\left\langle\phi_{3}\right|\right] } \\
& \otimes\left[F_{2}\left|\phi_{1}\right\rangle_{A B}^{S}\left\langle\phi_{1}\left|+\left(1-F_{2}\right)\right| \phi_{2}\right\rangle_{A B}^{S}\left\langle\phi_{2}\right|\right], \\
\rho_{C D}= & {\left[F_{1}\left|\phi_{1}\right\rangle_{C D}^{P}\left\langle\phi_{1}\left|+\left(1-F_{1}\right)\right| \phi_{3}\right\rangle_{C D}^{P}\left\langle\phi_{3}\right|\right] } \\
& \otimes\left[F_{2}\left|\phi_{1}\right\rangle_{C D}^{S}\left\langle\phi_{1}\left|+\left(1-F_{2}\right)\right| \phi_{2}\right\rangle_{C D}^{S}\left\langle\phi_{2}\right|\right] .
\end{aligned}
$$

Here the subscripts $A B$ and $C D$ represent two photon pairs shared by the two remote parties, called Alice and Bob, respectively. Photons $A$ and $C$ belong to Alice, and photons $B$ and $D$ belong to Bob. $F_{1}$ and $F_{2}$ represent the probabilities of states $\left|\phi_{1}\right\rangle_{A B}^{P}\left(\left|\phi_{1}\right\rangle_{C D}^{P}\right)$ and $\left|\phi_{1}\right\rangle_{A B}^{S}$ $\left(\left|\phi_{1}\right\rangle_{C D}^{S}\right)$ in the mixed states, respectively.

The initial state of the four-photon system $A B C D$ is $\rho_{0}=\rho_{A B} \otimes \rho_{C D}$. It can be viewed as the mixture of 16 maximally hyperentangled pure states. That is, it is the mixture of the four pure states in the polarization 


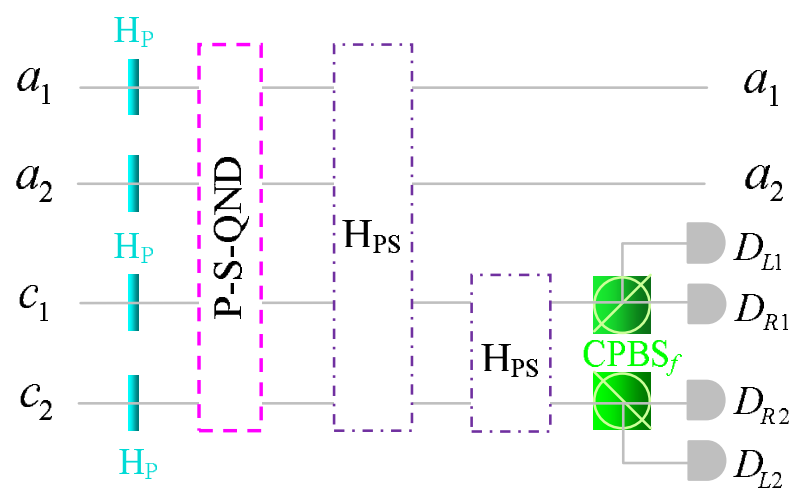

(a)

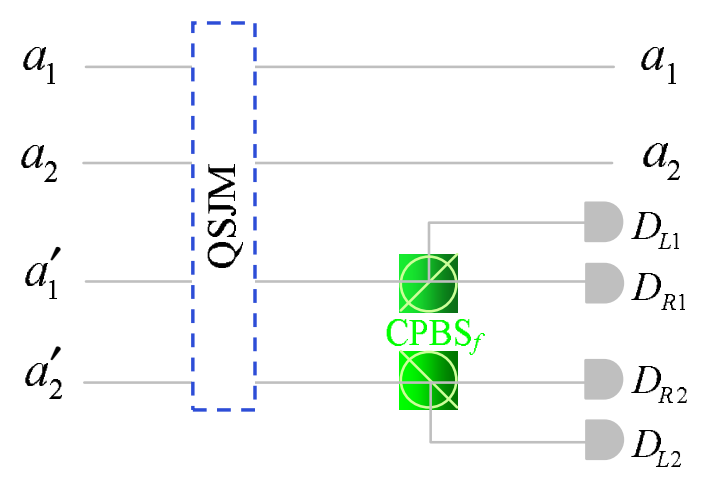

(b)

FIG. 4: (Color online) (a) Schematic diagram of the first step of our hyper-EPP for mixed hyperentangled Bell states with polarization bit-flip errors and spatial-mode phase-flip errors, resorting to the P-S-QND. Bob performs the same operations as Alice by replacing photons $A$ and $C$ with photons $B$ and $D$, respectively. (b) Schematic diagram of the second step of our hyper-EPP for mixed hyperentangled Bell states with polarization bit-flip errors and spatial-mode phase-flip errors, resorting to the QSJM. Bob performs the same operations as Alice by replacing photons $A$ and $A^{\prime}$ with photons $B$ and $B^{\prime}$, respectively. $D_{j}(j=L 1, R 1, R 2$, or $L 2)$ represents a single-photon detector.

DOF: $\left|\phi_{1}\right\rangle_{A B}^{P} \otimes\left|\phi_{1}\right\rangle_{C D}^{P}$ with the probability $F_{1}^{2},\left|\phi_{1}\right\rangle_{A B}^{P} \otimes$ $\left|\phi_{3}\right\rangle_{C D}^{P}$ with the probability $F_{1}\left(1-F_{1}\right),\left|\phi_{3}\right\rangle_{A B}^{P} \otimes\left|\phi_{1}\right\rangle_{C D}^{P}$ with the probability $F_{1}\left(1-F_{1}\right)$, and $\left|\phi_{3}\right\rangle_{A B}^{P} \otimes\left|\phi_{3}\right\rangle_{C D}^{P}$ with the probability $\left(1-F_{1}\right)^{2}$. It is also the mixture of the four pure states in the spatial-mode DOF: $\left|\phi_{1}\right\rangle_{A B}^{S} \otimes$ $\left|\phi_{1}\right\rangle_{C D}^{S}$ with the probability $F_{2}^{2},\left|\phi_{1}\right\rangle_{A B}^{S} \otimes\left|\phi_{2}\right\rangle_{C D}^{S}$ with the probability $F_{2}\left(1-F_{2}\right),\left|\phi_{2}\right\rangle_{A B}^{S} \otimes\left|\phi_{1}\right\rangle_{C D}^{S}$ with the probability $F_{2}\left(1-F_{2}\right)$, and $\left|\phi_{2}\right\rangle_{A B}^{S} \otimes\left|\phi_{2}\right\rangle_{C D}^{S}$ with the probability $\left(1-F_{2}\right)^{2}$.

The first step of our hyper-EPP for mixed hyperentangled Bell states with polarization bit-flip errors and spatial-mode phase-flip errors is shown in Fig 4(a), which requires Alice and Bob perform the Hadamard operations $H_{P}$ and P-S-QNDs on both the polarization and the spatial-mode DOFs of photon pairs $A C$ and $B D$ in sequence. After performing $H_{P S}$ on the polarization and the spatial-mode DOFs of photon pairs $A C$ and $B D, \mathrm{Al}-$ ice and Bob measure the states of the excess electron spins in QDs to read out the outcomes of the parity modes of photon pairs $A C$ and $B D$ in both the polarization and the spatial-mode DOFs.

(1) If the two photon pairs, $A C$ and $B D$, are in the same polarization parity mode and the same spatialmode parity mode (both in either the even-parity mode or the odd-parity mode), the polarization pure states $\left|\phi_{1}\right\rangle_{A B}^{P} \otimes\left|\phi_{1}\right\rangle_{C D}^{P}$ and $\left|\phi_{3}\right\rangle_{A B}^{P} \otimes\left|\phi_{3}\right\rangle_{C D}^{P}$ are distinguished from the other two polarization pure states, $\left|\phi_{1}\right\rangle_{A B}^{P} \otimes$ $\left|\phi_{3}\right\rangle_{C D}^{P}$ and $\left|\phi_{3}\right\rangle_{A B}^{P} \otimes\left|\phi_{1}\right\rangle_{C D}^{P}$, and the spatial-mode pure states $\left|\phi_{1}\right\rangle_{A B}^{S} \otimes\left|\phi_{1}\right\rangle_{C D}^{S}$ and $\left|\phi_{2}\right\rangle_{A B}^{S} \otimes\left|\phi_{2}\right\rangle_{C D}^{S}$ are distinguished from the other two spatial-mode pure states, $\left|\phi_{1}\right\rangle_{A B}^{S} \otimes\left|\phi_{2}\right\rangle_{C D}^{S}$ and $\left|\phi_{2}\right\rangle_{A B}^{S} \otimes\left|\phi_{1}\right\rangle_{C D}^{S}$. After the measurement on the excess electron spins in P-S-QNDs, the polarization DOF of the four-photon system is projected into a mixed state composed of states $\left|\Phi_{1}\right\rangle_{P}\left(\right.$ or $\left.\left|\Phi_{2}\right\rangle_{P}\right)$ and $\left|\Phi_{3}\right\rangle_{P}$ (or $\left|\Phi_{4}\right\rangle_{P}$ ), and the spatial-mode DOF of the four-photon system is projected into a mixed state composed of states $\left|\Phi_{1}\right\rangle_{S}$ (or $\left|\Phi_{2}\right\rangle_{S}$ ) and $\left|\Phi_{3}\right\rangle_{S}$ (or $\left|\Phi_{4}\right\rangle_{S}$ ). Here

$$
\begin{aligned}
\left|\Phi_{1}\right\rangle_{P} & =\frac{1}{\sqrt{2}}(|R R R R\rangle+|L L L L\rangle)_{A B C D}, \\
\left|\Phi_{2}\right\rangle_{P} & =\frac{1}{\sqrt{2}}(|R R L L\rangle+|L L R R\rangle)_{A B C D}, \\
\left|\Phi_{3}\right\rangle_{P} & =\frac{1}{\sqrt{2}}(|R L R L\rangle+|L R L R\rangle)_{A B C D}, \\
\left|\Phi_{4}\right\rangle_{P} & =\frac{1}{\sqrt{2}}(|R L L R\rangle+|L R R L\rangle)_{A B C D}, \\
\left|\Phi_{1}\right\rangle_{S} & =\frac{1}{\sqrt{2}}\left(\left|a_{1} b_{1} c_{1} d_{1}\right\rangle+\left|a_{2} b_{2} c_{2} d_{2}\right\rangle\right), \\
\left|\Phi_{2}\right\rangle_{S} & =\frac{1}{\sqrt{2}}\left(\left|a_{1} b_{1} c_{2} d_{2}\right\rangle+\left|a_{2} b_{2} c_{1} d_{1}\right\rangle\right), \\
\left|\Phi_{3}\right\rangle_{S} & =\frac{1}{\sqrt{2}}\left(\left|a_{1} b_{2} c_{1} d_{2}\right\rangle+\left|a_{2} b_{1} c_{2} d_{1}\right\rangle\right), \\
\left|\Phi_{4}\right\rangle_{S} & =\frac{1}{\sqrt{2}}\left(\left|a_{1} b_{2} c_{2} d_{1}\right\rangle+\left|a_{2} b_{1} c_{1} d_{2}\right\rangle\right) .
\end{aligned}
$$

State $\left|\Phi_{2}\right\rangle_{P}$ can be transformed into $\left|\Phi_{1}\right\rangle_{P}$ by performing the polarization bit-flip operations $\sigma_{x}^{P}$ on both photons $C$ and $D$. In a similar way, states $\left|\Phi_{4}\right\rangle_{P},\left|\Phi_{2}\right\rangle_{S}$, and $\left|\Phi_{4}\right\rangle_{S}$ can be transformed into states $\left|\Phi_{3}\right\rangle_{P},\left|\Phi_{1}\right\rangle_{S}$, and $\left|\Phi_{3}\right\rangle_{S}$, respectively. Subsequently, Alice and Bob perform the Hadamard operations on both the polarization and the spatial-mode DOFs of photons $C$ and $D$, and states $\left|\Phi_{1}\right\rangle_{P},\left|\Phi_{3}\right\rangle_{P},\left|\Phi_{1}\right\rangle_{S}$, and $\left|\Phi_{3}\right\rangle_{S}$ are transformed into states $\left|\Phi_{1}^{\prime}\right\rangle_{P},\left|\Phi_{3}^{\prime}\right\rangle_{P},\left|\Phi_{1}^{\prime}\right\rangle_{S}$, and $\left|\Phi_{3}^{\prime}\right\rangle_{S}$, respectively. Here

$$
\begin{aligned}
\left|\Phi_{1}^{\prime}\right\rangle_{P}= & \frac{1}{2 \sqrt{2}}\left[(|R R\rangle+|L L\rangle)_{A B}(|R R\rangle+|L L\rangle)_{C D}\right. \\
& \left.+(|R R\rangle-|L L\rangle)_{A B}(|R L\rangle+|L R\rangle)_{C D}\right], \\
\left|\Phi_{3}^{\prime}\right\rangle_{P}= & \frac{1}{2 \sqrt{2}}\left[(|R L\rangle+|L R\rangle)_{A B}(|R R\rangle-|L L\rangle)_{C D}\right.
\end{aligned}
$$




$$
\begin{aligned}
& \left.+(-|R L\rangle+|L R\rangle)_{A B}(|R L\rangle-|L R\rangle)_{C D}\right] \\
\left|\Phi_{1}^{\prime}\right\rangle_{S}= & \frac{1}{2 \sqrt{2}}\left[\left(\left|a_{1} b_{1}\right\rangle+\left|a_{2} b_{2}\right\rangle\right)\left(\left|c_{1} d_{1}\right\rangle+\left|c_{2} d_{2}\right\rangle\right)\right. \\
& \left.+\left(\left|a_{1} b_{1}\right\rangle-\left|a_{2} b_{2}\right\rangle\right)\left(\left|c_{1} d_{2}\right\rangle+\left|c_{2} d_{1}\right\rangle\right)\right] \\
\left|\Phi_{3}^{\prime}\right\rangle_{S}= & \frac{1}{2 \sqrt{2}}\left[\left(\left|a_{1} b_{2}\right\rangle+\left|a_{2} b_{1}\right\rangle\right)\left(\left|c_{1} d_{1}\right\rangle-\left|c_{2} d_{2}\right\rangle\right)\right. \\
& \left.+\left(-\left|a_{1} b_{2}\right\rangle+\left|a_{2} b_{1}\right\rangle\right)\left(\left|c_{1} d_{2}\right\rangle-\left|c_{2} d_{1}\right\rangle\right)\right]
\end{aligned}
$$

Finally, photons $C$ and $D$ are detected with single-photon detectors, shown in Fig 4 (a). If the two clicked singlephoton detectors of photons $C$ and $D$ are in the evenparity spatial mode (even-parity polarization mode), the spatial-mode (polarization) DOF of the two-photon system $A B$ is projected into state $\left|\phi_{1}\right\rangle_{A B}^{S}$ or $\left|\phi_{3}\right\rangle_{A B}^{S}\left(\left|\phi_{1}\right\rangle_{A B}^{P}\right.$ or $\left.\left|\phi_{3}\right\rangle_{A B}^{P}\right)$. If the outcome of the two clicked detectors is in the odd-parity spatial mode (odd-parity polarization mode), a phase-flip operation $\sigma_{z}^{S}=\left|b_{1}\right\rangle\left\langle b_{1}|-| b_{2}\right\rangle\left\langle b_{2}\right|$ $\left(\sigma_{z}^{P}\right)$ on the spatial-mode (polarization) DOF of photon $B$ is required to obtain state $\left|\phi_{1}\right\rangle_{A B}^{S}$ or $\left|\phi_{3}\right\rangle_{A B}^{S}\left(\left|\phi_{1}\right\rangle_{A B}^{P}\right.$ or $\left.\left|\phi_{3}\right\rangle_{A B}^{P}\right)$.

(2) If photon pairs $A C$ and $B D$ are in different polarization parity modes and different spatial-mode parity modes (one pair is in the even-parity mode and the other is in the odd-parity mode), the polarization pure states $\left|\phi_{1}\right\rangle_{A B}^{P} \otimes\left|\phi_{3}\right\rangle_{C D}^{P}$ and $\left|\phi_{3}\right\rangle_{A B}^{P} \otimes\left|\phi_{1}\right\rangle_{C D}^{P}$ are distinguished from the other two polarization pure states, $\left|\phi_{1}\right\rangle_{A B}^{P} \otimes\left|\phi_{1}\right\rangle_{C D}^{P}$ and $\left|\phi_{3}\right\rangle_{A B}^{P} \otimes\left|\phi_{3}\right\rangle_{C D}^{P}$, and the spatialmode pure states $\left|\phi_{1}\right\rangle_{A B}^{S} \otimes\left|\phi_{2}\right\rangle_{C D}^{S}$ and $\left|\phi_{2}\right\rangle_{A B}^{S} \otimes\left|\phi_{1}\right\rangle_{C D}^{S}$ are distinguished from the other two spatial-mode pure states, $\left|\phi_{1}\right\rangle_{A B}^{S} \otimes\left|\phi_{1}\right\rangle_{C D}^{S}$ and $\left|\phi_{2}\right\rangle_{A B}^{S} \otimes\left|\phi_{2}\right\rangle_{C D}^{S}$, respectively. After the measurement on the excess electron spins in P-S-QNDs, the polarization DOF of the fourphoton system is projected into a mixed state composed of states $\left|\Phi_{5}\right\rangle_{P}$ (or $\left.\left|\Phi_{6}\right\rangle_{P}\right)$ and $\left|\Phi_{7}\right\rangle_{P}\left(\right.$ or $\left.\left|\Phi_{8}\right\rangle_{P}\right)$, and the spatial-mode DOF of the four-photon system is projected into a mixed state composed of states $\left|\Phi_{5}\right\rangle_{S}\left(\right.$ or $\left.\left|\Phi_{6}\right\rangle_{S}\right)$ and $\left|\Phi_{7}\right\rangle_{S}\left(\right.$ or $\left.\left|\Phi_{8}\right\rangle_{S}\right)$. Here

$$
\begin{aligned}
\left|\Phi_{5}\right\rangle_{P} & =\frac{1}{\sqrt{2}}(|R R R L\rangle+|L L L R\rangle)_{A B C D} \\
\left|\Phi_{6}\right\rangle_{P} & =\frac{1}{\sqrt{2}}(|R R L R\rangle+|L L R L\rangle)_{A B C D} \\
\left|\Phi_{7}\right\rangle_{P} & =\frac{1}{\sqrt{2}}(|R L R R\rangle+|L R L L\rangle)_{A B C D} \\
\left|\Phi_{8}\right\rangle_{P} & =\frac{1}{\sqrt{2}}(|R L L L\rangle+|L R R R\rangle)_{A B C D} \\
\left|\Phi_{5}\right\rangle_{S} & =\frac{1}{\sqrt{2}}\left(\left|a_{1} b_{1} c_{1} d_{2}\right\rangle+\left|a_{2} b_{2} c_{2} d_{1}\right\rangle\right) \\
\left|\Phi_{6}\right\rangle_{S} & =\frac{1}{\sqrt{2}}\left(\left|a_{1} b_{1} c_{2} d_{1}\right\rangle+\left|a_{2} b_{2} c_{1} d_{2}\right\rangle\right) \\
\left|\Phi_{7}\right\rangle_{S} & =\frac{1}{\sqrt{2}}\left(\left|a_{1} b_{2} c_{1} d_{1}\right\rangle+\left|a_{2} b_{1} c_{2} d_{2}\right\rangle\right) \\
\left|\Phi_{8}\right\rangle_{S} & =\frac{1}{\sqrt{2}}\left(\left|a_{1} b_{2} c_{2} d_{2}\right\rangle+\left|a_{2} b_{1} c_{1} d_{1}\right\rangle\right) .
\end{aligned}
$$

As Alice and Bob cannot identify which photon pair, $A B$ or $C D$, has the polarization bit-flip error (the spatialmode phase-flip error), they have to discard the two photon pairs in this case.

(3) If photon pairs $A C$ and $B D$ are in the same polarization parity mode and different spatial-mode parity modes, the polarization pure states $\left|\phi_{1}\right\rangle_{A B}^{P} \otimes\left|\phi_{1}\right\rangle_{C D}^{P}$ and $\left|\phi_{3}\right\rangle_{A B}^{P} \otimes\left|\phi_{3}\right\rangle_{C D}^{P}$ are distinguished from the other two polarization pure states, $\left|\phi_{1}\right\rangle_{A B}^{P} \otimes\left|\phi_{3}\right\rangle_{C D}^{P}$ and $\left|\phi_{3}\right\rangle_{A B}^{P} \otimes$ $\left|\phi_{1}\right\rangle_{C D}^{P}$, and the spatial-mode pure states $\left|\phi_{1}\right\rangle_{A B}^{S} \otimes\left|\phi_{2}\right\rangle_{C D}^{S}$ and $\left|\phi_{2}\right\rangle_{A B}^{S} \otimes\left|\phi_{1}\right\rangle_{C D}^{S}$ are distinguished from the other two spatial-mode pure states, $\left|\phi_{1}\right\rangle_{A B}^{S} \otimes\left|\phi_{1}\right\rangle_{C D}^{S}$ and $\left|\phi_{2}\right\rangle_{A B}^{S} \otimes$ $\left|\phi_{2}\right\rangle_{C D}^{S}$. After the measurement on the excess electron spins in P-S-QNDs, the polarization DOF of the fourphoton system is projected into a mixed state composed of states $\left|\Phi_{1}\right\rangle_{P}$ (or $\left.\left|\Phi_{2}\right\rangle_{P}\right)$ and $\left|\Phi_{3}\right\rangle_{P}\left(\right.$ or $\left.\left|\Phi_{4}\right\rangle_{P}\right)$, and the spatial-mode DOF of the four-photon system is projected into a mixed state composed of states $\left|\Phi_{5}\right\rangle_{S}\left(\right.$ or $\left.\left|\Phi_{6}\right\rangle_{S}\right)$ and $\left|\Phi_{7}\right\rangle_{S}\left(\right.$ or $\left.\left|\Phi_{8}\right\rangle_{S}\right)$. In this case, the second step is required in our hyper-EPP with QSJM.

(4) If photon pairs $A C$ and $B D$ are in different polarization parity modes and the same spatial-mode parity mode, the polarization pure states $\left|\phi_{1}\right\rangle_{A B}^{P} \otimes\left|\phi_{3}\right\rangle_{C D}^{P}$ and $\left|\phi_{3}\right\rangle_{A B}^{P} \otimes\left|\phi_{1}\right\rangle_{C D}^{P}$ are distinguished from the other two polarization pure states, $\left|\phi_{1}\right\rangle_{A B}^{P} \otimes\left|\phi_{1}\right\rangle_{C D}^{P}$ and $\left|\phi_{3}\right\rangle_{A B}^{P} \otimes$ $\left|\phi_{3}\right\rangle_{C D}^{P}$, and the spatial-mode pure states $\left|\phi_{1}\right\rangle_{A B}^{S} \otimes\left|\phi_{1}\right\rangle_{C D}^{S}$ and $\left|\phi_{2}\right\rangle_{A B}^{S} \otimes\left|\phi_{2}\right\rangle_{C D}^{S}$ are distinguished from the other two spatial-mode pure states, $\left|\phi_{1}\right\rangle_{A B}^{S} \otimes\left|\phi_{2}\right\rangle_{C D}^{S}$ and $\left|\phi_{2}\right\rangle_{A B}^{S} \otimes$ $\left|\phi_{1}\right\rangle_{C D}^{S}$. After the measurement on the excess electron spins in P-S-QNDs, the polarization DOF of the fourphoton system is projected into a mixed state composed of states $\left|\Phi_{5}\right\rangle_{P}\left(\right.$ or $\left.\left|\Phi_{6}\right\rangle_{P}\right)$ and $\left|\Phi_{7}\right\rangle_{P}\left(\right.$ or $\left.\left|\Phi_{8}\right\rangle_{P}\right)$, and the spatial-mode DOF of the four-photon system is projected into a mixed state composed of states $\left|\Phi_{1}\right\rangle_{S}$ (or $\left|\Phi_{2}\right\rangle_{S}$ ) and $\left|\Phi_{3}\right\rangle_{S}$ (or $\left|\Phi_{4}\right\rangle_{S}$ ). In this case, the second step is required in our hyper-EPP with QSJM as well.

\section{B. The second step of our two-step hyper-EPP with QSJM}

In this step, we show that the efficiency of the hyperEPP is improved with QSJM by preserving cases (3) and (4) in the first step, as shown in Fig. 4(b).

Suppose that there are four identical photon pairs, $A B$, $C D, A^{\prime} B^{\prime}$, and $C^{\prime} D^{\prime}$, shared by Alice and Bob. Photons $A, C, A^{\prime}, C^{\prime}$ belong to Alice, and photons $B, D, B^{\prime}, D^{\prime}$ belong to Bob. Alice and Bob perform the same operations on the four-photon systems $A B C D$ and $A^{\prime} B^{\prime} C^{\prime} D^{\prime}$ as they did in the first step. If the four-photon systems $A B C D$ and $A^{\prime} B^{\prime} C^{\prime} D^{\prime}$ are projected into the states in cases (4) and (3) in the first step, respectively, Alice and Bob can use the QSJM (introduced in Sec. IIB) to transfer the polarization state of the four-photon system $A^{\prime} B^{\prime} C^{\prime} D^{\prime}$ to the polarization state of the four-photon system $A B C D$ (discussed in Appendix $\mathrm{A}$ ). The polarization state of the four-photon system ABCD is in a mixed state composed of states $\left|\Phi_{1}\right\rangle_{P}$ and $\left|\Phi_{3}\right\rangle_{P}$, and the spatial-mode DOF of the four-photon system ABCD 
is in a mixed state composed of states $\left|\Phi_{1}\right\rangle_{S}$ and $\left|\Phi_{3}\right\rangle_{S}$, which is the preserving condition of case (1) in the first step. After Hadamard operations and detections are performed on both the polarization and spatial-mode DOFs of photons $C$ and $D$ and the conditional phase-flip operation $\sigma_{z}^{S}\left(\sigma_{z}^{P}\right)$ is performed on photon $B$, the spatial-mode states $\left|\phi_{1}\right\rangle_{A B}^{S}$ and $\left|\phi_{3}\right\rangle_{A B}^{S}$ (polarization states $\left|\phi_{1}\right\rangle_{A B}^{P}$ and $\left.\left|\phi_{3}\right\rangle_{A B}^{P}\right)$ are obtained.

If the four-photon systems $A B C D$ and $A^{\prime} B^{\prime} C^{\prime} D^{\prime}$ are projected into the states in cases (3) and (4) in the first step, respectively, Alice and Bob can use the QSJM to transfer the spatial-mode state of the four-photon system $A^{\prime} B^{\prime} C^{\prime} D^{\prime}$ to the spatial-mode state of the four-photon system $A B C D$. Then the preserving condition of case (1) in the first step is achieved. After Hadamard operations and detections are performed on both the polarization and spatial-mode DOFs of photons $C$ and $D$ and the conditional phase-flip operation $\sigma_{z}^{S}\left(\sigma_{z}^{P}\right)$ is performed on photon $B$, the spatial-mode states $\left|\phi_{1}\right\rangle_{A B}^{S}$ and $\left|\phi_{3}\right\rangle_{A B}^{S}$ (polarization states $\left|\phi_{1}\right\rangle_{A B}^{P}$ and $\left|\phi_{3}\right\rangle_{A B}^{P}$ ) are obtained.

After the first round of our hyper-EPP process with these two steps, the state of the photon pair $A B$ is transformed into

$$
\begin{aligned}
\rho_{A B}^{\prime}= & {\left[F_{1}^{\prime}\left|\phi_{1}\right\rangle_{A B}^{P}\left\langle\phi_{1}\left|+\left(1-F_{1}^{\prime}\right)\right| \phi_{3}\right\rangle_{A B}^{P}\left\langle\phi_{3}\right|\right] } \\
& \otimes\left[F_{2}^{\prime}\left|\phi_{1}\right\rangle_{A B}^{S}\left\langle\phi_{1}\left|+\left(1-F_{2}^{\prime}\right)\right| \phi_{3}\right\rangle_{A B}^{S}\left\langle\phi_{3}\right|\right] .
\end{aligned}
$$

Here $F_{1}^{\prime}=\frac{F_{1}^{2}}{\left[F_{1}^{2}+\left(1-F_{1}\right)^{2}\right]}, F_{2}^{\prime}=\frac{F_{2}^{2}}{\left[F_{2}^{2}+\left(1-F_{2}\right)^{2}\right]}$, and $F_{i}>1 / 2$ $(i=1,2)$. The fidelity of state $\left|\phi_{1}\right\rangle_{A B}^{P} \otimes\left|\phi_{1}\right\rangle_{A B}^{S}$ in Eq. (16) is $F^{\prime}=F_{1}^{\prime} \times F_{2}^{\prime}$. State $\left|\phi_{3}\right\rangle_{A B}^{S}$ can be transformed into $\left|\phi_{2}\right\rangle_{A B}^{S}$ with the Hadamard operations on the spatial-mode DOF of photons $A$ and $B$. With the iteration of our hyper-EPP process, the fidelity of the two-photon state can be improved (shown in Fig. 5 for the cases with $F_{1}=F_{2}$ ).

The efficiency of an EPP is defined as the probability of obtaining a high-fidelity entangled photon system from a pair of photon systems transmitted over a noisy channel without photon loss. Our hyper-EPP is constructed to purify the mixed hyperentangled Bell states with spatialmode phase-flip errors and polarization bit-flip errors. In our previous work [46], we introduced a hyper-EPP for the mixed hyperentangled Bell states with bit-flip errors in both the spatial-mode and polarization DOFs, which only preserves case (1) in the first step with the probability of

$$
\left[F_{1}^{2}+\left(1-F_{1}\right)^{2}\right]\left[F_{2}^{2}+\left(1-F_{2}\right)^{2}\right]
$$

(in the first round of the hyper-EPP process), and the efficiency is

$$
Y_{0}=\left[F_{1}^{2}+\left(1-F_{1}\right)^{2}\right]\left[F_{2}^{2}+\left(1-F_{2}\right)^{2}\right] .
$$

In the present hyper-EPP, cases (3) and (4) in the first step can also be preserved with our QSJM, and the efficiency of the first round of the hyper-EPP process is

$$
Y=F_{2}^{2}+\left(1-F_{2}\right)^{2}
$$

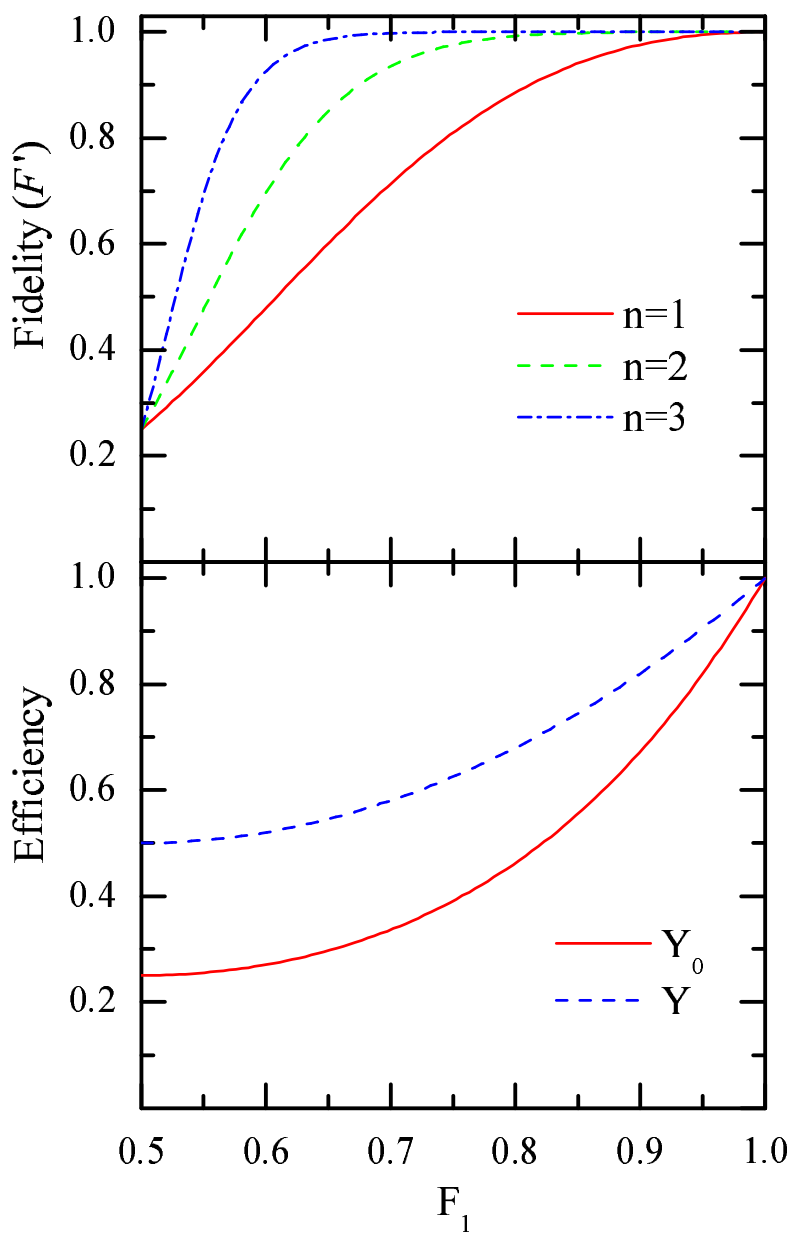

FIG. 5: (Color online) The fidelity and efficiency of our hyperEPP for mixed hyperentangled Bell states. $F^{\prime}=F_{1}^{\prime} \times F_{2}^{\prime} \cdot n$ is the iteration number of the hyper-EPP process. $Y_{0}$ and $Y$ represent the efficiencies of the first round of the hyperEPP processes without and with QSJM, respectively. The parameters of the mixed hyperentangled Bell state are chosen to be $F_{1}=F_{2}$.

when $F_{1}>F_{2}$. The efficiency of the hyper-EPP is improved by resorting to the QSJM (shown in Fig. 5 for the cases with $F_{1}=F_{2}$ ).

\section{HYPER-EPP FOR HYPERENTANGLED GHZ STATES WITH QSJM}

We can generalize our hyper-EPP for mixed hyperentangled GHZ states of three-photon systems. There are eight polarization GHZ states and eight spatial-mode GHZ states, and they are described as follows:

$$
\begin{aligned}
\left|\psi_{0}^{ \pm}\right\rangle_{P} & =\frac{1}{\sqrt{2}}(|R R R\rangle \pm|L L L\rangle)_{A B C} \\
\left|\psi_{1}^{ \pm}\right\rangle_{P} & =\frac{1}{\sqrt{2}}(|R R L\rangle \pm|L L R\rangle)_{A B C}
\end{aligned}
$$




$$
\begin{aligned}
\left|\psi_{2}^{ \pm}\right\rangle_{P} & =\frac{1}{\sqrt{2}}(|R L R\rangle \pm|L R L\rangle)_{A B C}, \\
\left|\psi_{3}^{ \pm}\right\rangle_{P} & =\frac{1}{\sqrt{2}}(|L R R\rangle \pm|R L L\rangle)_{A B C}, \\
\left|\psi_{0}^{ \pm}\right\rangle_{S} & =\frac{1}{\sqrt{2}}\left(\left|a_{1} b_{1} c_{1}\right\rangle \pm\left|a_{2} b_{2} c_{2}\right\rangle\right), \\
\left|\psi_{1}^{ \pm}\right\rangle_{S} & =\frac{1}{\sqrt{2}}\left(\left|a_{1} b_{1} c_{2}\right\rangle \pm\left|a_{2} b_{2} c_{1}\right\rangle\right), \\
\left|\psi_{2}^{ \pm}\right\rangle_{S} & =\frac{1}{\sqrt{2}}\left(\left|a_{1} b_{2} c_{1}\right\rangle \pm\left|a_{2} b_{1} c_{2}\right\rangle\right), \\
\left|\psi_{3}^{ \pm}\right\rangle_{S} & =\frac{1}{\sqrt{2}}\left(\left|a_{2} b_{1} c_{1}\right\rangle \pm\left|a_{1} b_{2} c_{2}\right\rangle\right) .
\end{aligned}
$$

Here the subscripts $A, B$, and $C$ represent the photons obtained by the remote users Alice, Bob, and Charlie, respectively. Suppose that the original state of the threephoton system $A B C$ is $\left|\psi_{0}^{+}\right\rangle_{P} \otimes\left|\psi_{0}^{+}\right\rangle_{S}$. If there is a polarization bit-flip error on the original state of the threephoton system after its transmission over a noisy channel, the polarization state of the three-photon system will become $\left|\psi_{1}^{+}\right\rangle_{P},\left|\psi_{2}^{+}\right\rangle_{P}$, or $\left|\psi_{3}^{+}\right\rangle_{P}$. However, a spatial-mode phase-flip error is more likely to take place on the original state of the three-photon system [46, 61], and the spatial-mode state of the three-photon system will become $\left|\psi_{0}^{-}\right\rangle_{S}$.

After the photon system is transmitted over the noisy channel with polarization bit-flip errors and spatial-mode phase-flip errors, its state becomes

$$
\begin{aligned}
\rho= & \left(F_{0}\left|\psi_{0}^{+}\right\rangle_{P}\left\langle\psi_{0}^{+}\left|+F_{1}\right| \psi_{1}^{+}\right\rangle_{P}\left\langle\psi_{1}^{+}\right|\right. \\
& \left.+F_{2}\left|\psi_{2}^{+}\right\rangle_{P}\left\langle\psi_{2}^{+}\left|+F_{3}\right| \psi_{3}^{+}\right\rangle_{P}\left\langle\psi_{3}^{+}\right|\right) \\
& \otimes\left(P_{0}\left|\psi_{0}^{+}\right\rangle_{S}\left\langle\psi_{0}^{+}\left|+P_{1}\right| \psi_{0}^{-}\right\rangle_{S}\left\langle\psi_{0}^{-}\right|\right)
\end{aligned}
$$

Here $F_{0}$ and $P_{0}$ are the probabilities of states $\left|\psi_{0}^{+}\right\rangle_{P}$ and $\left|\psi_{0}^{+}\right\rangle_{S}$ in the mixed states, respectively, and they satisfy the relations $F_{0}+F_{1}+F_{2}+F_{3}=1$ and $P_{0}+P_{1}=1$. In order to obtain high-fidelity entangled three-photon systems, the three remote users, Alice, Bob, and Charlie, have to perform hyper-EPP on the three-photon systems, which requires them to divide their photon systems into many groups with a pair of three-photon systems in each group. The photons in each group are labeled $A B C A^{\prime} B^{\prime} C^{\prime}$, where $A B C$ and $A^{\prime} B^{\prime} C^{\prime}$ represent two identical three-photon systems in the same mixed hyperentangled GHZ state. As the phase-flip error of the three-photon GHZ state cannot be transformed into the bit-flip error with Hadamard operations, we discuss the principles of the EPPs for the polarization states and the spatial-mode states of the three-photon systems in Secs. IVA and IV B, respectively. The hyper-EPP for mixed hyperentangled GHZ states with our QSJM is introduced in Sec. IV C.

\section{A. EPP for polarization GHZ states}

The polarization state of system $A B C A^{\prime} B^{\prime} C^{\prime}$ can be viewed as the mixture of 16 pure states $\left|\psi_{i}^{+}\right\rangle_{P} \otimes\left|\psi_{j}^{+}\right\rangle_{P}$ with the probability of $F_{i} F_{j}(i, j=0,1,2,3)$. Alice, Bob, and Charlie perform polarization parity-check QNDs on their photon pairs $A A^{\prime}, B B^{\prime}$, and $C C^{\prime}$, respectively, and they pick the groups in which the three photon pairs are all in the even-parity polarization mode or all in the oddparity polarization mode. If the polarization states of the three photon pairs are all in the even-parity mode, the polarization DOF of the six-photon system will project into a mixed state composed of the four pure states $\left|\Psi_{0}\right\rangle_{P}$, $\left|\Psi_{1}\right\rangle_{P},\left|\Psi_{2}\right\rangle_{P}$, and $\left|\Psi_{3}\right\rangle_{P}$. Here

$$
\begin{aligned}
\left|\Psi_{0}\right\rangle_{P} & =\frac{1}{\sqrt{2}}(|R R R\rangle|R R R\rangle+|L L L\rangle|L L L\rangle)_{A B C A^{\prime} B^{\prime} C^{\prime}}, \\
\left|\Psi_{1}\right\rangle_{P} & =\frac{1}{\sqrt{2}}(|R R L\rangle|R R L\rangle+|L L R\rangle|L L R\rangle)_{A B C A^{\prime} B^{\prime} C^{\prime}}, \\
\left|\Psi_{2}\right\rangle_{P} & =\frac{1}{\sqrt{2}}(|R L R\rangle|R L R\rangle+|L R L\rangle|L R L\rangle)_{A B C A^{\prime} B^{\prime} C^{\prime}}, \\
\left|\Psi_{3}\right\rangle_{P} & =\frac{1}{\sqrt{2}}(|L R R\rangle|L R R\rangle+|R L L\rangle|R L L\rangle)_{A B C A^{\prime} B^{\prime} C^{\prime}} .
\end{aligned}
$$

If the polarization states of the three photon pairs are all in the odd-parity mode, the polarization DOF of the six-photon system will project into a mixed state composed of the four pure states $\left|\Psi_{0}^{\prime}\right\rangle_{P},\left|\Psi_{1}^{\prime}\right\rangle_{P},\left|\Psi_{2}^{\prime}\right\rangle_{P}$, and $\left|\Psi_{3}^{\prime}\right\rangle_{P}$. Here

$$
\begin{aligned}
\left|\Psi_{0}^{\prime}\right\rangle_{P} & =\frac{1}{\sqrt{2}}(|R R R\rangle|L L L\rangle+|L L L\rangle|R R R\rangle)_{A B C A^{\prime} B^{\prime} C^{\prime}}, \\
\left|\Psi_{1}^{\prime}\right\rangle_{P} & =\frac{1}{\sqrt{2}}(|R R L\rangle|L L R\rangle+|L L R\rangle|R R L\rangle)_{A B C A^{\prime} B^{\prime} C^{\prime}}, \\
\left|\Psi_{2}^{\prime}\right\rangle_{P} & =\frac{1}{\sqrt{2}}(|R L R\rangle|L R L\rangle+|L R L\rangle|R L R\rangle)_{A B C A^{\prime} B^{\prime} C^{\prime}}, \\
\left|\Psi_{3}^{\prime}\right\rangle_{P} & =\frac{1}{\sqrt{2}}(|L R R\rangle|R L L\rangle+|R L L\rangle|L R R\rangle)_{A B C A^{\prime} B^{\prime} C^{\prime}} .
\end{aligned}
$$

With the polarization bit-flip operations $\sigma_{x}^{P}$ on photons $A^{\prime}, B^{\prime}$ and $C^{\prime}$, the polarization state $\left|\Psi_{i}^{\prime}\right\rangle_{P}$ can be transformed into $\left|\Psi_{i}\right\rangle_{P}(i=0,1,2,3)$. After Hardmard operations and detections are performed on the polarization DOF of photons $A^{\prime}, B^{\prime}$ and $C^{\prime}$ and the conditional phase-flip operation $\sigma_{z}^{P}$ is performed on photon $A$ [43], states $\left|\psi_{0}^{+}\right\rangle_{P},\left|\psi_{1}^{+}\right\rangle_{P},\left|\psi_{2}^{+}\right\rangle_{P}$, and $\left|\psi_{3}^{+}\right\rangle_{P}$ are obtained with the probabilities $F_{0}^{2}, F_{1}^{2}, F_{2}^{2}$, and $F_{3}^{2}$, respectively. If the results of the three polarization parity-check QNDs have other outcomes, which of the three-photon systems, $A B C$ or $A^{\prime} B^{\prime} C^{\prime}$, has the polarization bit-flip error is ambiguous, which will be discussed in the hyper-EPP with our QSJM in Sec. IVC. 


\section{B. EPP for spatial-mode GHZ states}

The spatial-mode state of system $A B C A^{\prime} B^{\prime} C^{\prime}$ can be viewed as the mixture of four pure states $\left|\psi_{0}^{+}\right\rangle_{S} \otimes\left|\psi_{0}^{+}\right\rangle_{S}$, $\left|\psi_{0}^{+}\right\rangle_{S} \otimes\left|\psi_{0}^{-}\right\rangle_{S},\left|\psi_{0}^{-}\right\rangle_{S} \otimes\left|\psi_{0}^{+}\right\rangle_{S}$, and $\left|\psi_{0}^{-}\right\rangle_{S} \otimes\left|\psi_{0}^{-}\right\rangle_{S}$, with the probabilities of $P_{0}^{2}, P_{0} P_{1}, P_{0} P_{1}$, and $P_{1}^{2}$, respectively. With spatial-mode Hadamard operations on the three photons in each subsystem, states $\left|\psi_{0}^{+}\right\rangle_{S}$ and $\left|\psi_{0}^{-}\right\rangle_{S}$ are transformed into states $\left|\varphi^{+}\right\rangle_{S}$ and $\left|\varphi^{-}\right\rangle_{S}$, respectively. Here

$$
\begin{aligned}
\left|\varphi^{+}\right\rangle_{S} & =\frac{1}{2}\left(\left|a_{1} b_{1} c_{1}\right\rangle+\left|a_{1} b_{2} c_{2}\right\rangle+\left|a_{2} b_{2} c_{1}\right\rangle+\left|a_{2} b_{1} c_{2}\right\rangle\right), \\
\left|\varphi^{-}\right\rangle_{S} & =\frac{1}{2}\left(\left|a_{1} b_{1} c_{2}\right\rangle+\left|a_{1} b_{2} c_{1}\right\rangle+\left|a_{2} b_{2} c_{2}\right\rangle+\left|a_{2} b_{1} c_{1}\right\rangle\right) .
\end{aligned}
$$

Alice, Bob, and Charlie perform spatial-mode paritycheck QNDs on their photon pairs $A A^{\prime}, B B^{\prime}$, and $C C^{\prime}$, respectively, and they pick the groups with an odd number of photon pairs in the even-parity spatial mode.

If the three photon pairs are all in the even-parity spatial mode, the spatial-mode DOF of the six-photon system is projected into a mixed state composed of the two pure states $\left|\Psi_{0}\right\rangle_{S}$ and $\left|\Psi_{1}\right\rangle_{S}$. Here

$$
\begin{aligned}
\left|\Psi_{0}\right\rangle_{S}= & \frac{1}{2}\left(\left|a_{1} b_{1} c_{1}\right\rangle\left|a_{1}^{\prime} b_{1}^{\prime} c_{1}^{\prime}\right\rangle+\left|a_{1} b_{2} c_{2}\right\rangle\left|a_{1}^{\prime} b_{2}^{\prime} c_{2}^{\prime}\right\rangle\right. \\
& \left.+\left|a_{2} b_{2} c_{1}\right\rangle\left|a_{2}^{\prime} b_{2}^{\prime} c_{1}^{\prime}\right\rangle+\left|a_{2} b_{1} c_{2}\right\rangle\left|a_{2}^{\prime} b_{1}^{\prime} c_{2}^{\prime}\right\rangle\right), \\
\left|\Psi_{1}\right\rangle_{S}= & \frac{1}{2}\left(\left|a_{1} b_{1} c_{2}\right\rangle\left|a_{1}^{\prime} b_{1}^{\prime} c_{2}^{\prime}\right\rangle+\left|a_{1} b_{2} c_{1}\right\rangle\left|a_{1}^{\prime} b_{2}^{\prime} c_{1}^{\prime}\right\rangle\right. \\
& \left.+\left|a_{2} b_{2} c_{2}\right\rangle\left|a_{2}^{\prime} b_{2}^{\prime} c_{2}^{\prime}\right\rangle+\left|a_{2} b_{1} c_{1}\right\rangle\left|a_{2}^{\prime} b_{1}^{\prime} c_{1}^{\prime}\right\rangle\right) .
\end{aligned}
$$

If photon pairs $A A^{\prime}$ and $B B^{\prime}$ are in the odd-parity spatial mode and photon pair $C C^{\prime}$ is in the even-parity spatial mode, the spatial-mode DOF of the six-photon system is projected into a mixed state composed of the two pure states $\left|\Psi_{0}^{\prime}\right\rangle_{S}$ and $\left|\Psi_{1}^{\prime}\right\rangle_{S}$. Here

$$
\begin{aligned}
\left|\Psi_{0}^{\prime}\right\rangle_{S}= & \frac{1}{2}\left(\left|a_{1} b_{1} c_{1}\right\rangle\left|a_{2}^{\prime} b_{2}^{\prime} c_{1}^{\prime}\right\rangle+\left|a_{2} b_{2} c_{1}\right\rangle\left|a_{1}^{\prime} b_{1}^{\prime} c_{1}^{\prime}\right\rangle\right. \\
& \left.+\left|a_{1} b_{2} c_{2}\right\rangle\left|a_{2}^{\prime} b_{1}^{\prime} c_{2}^{\prime}\right\rangle+\left|a_{2} b_{1} c_{2}\right\rangle\left|a_{1}^{\prime} b_{2}^{\prime} c_{2}^{\prime}\right\rangle\right), \\
\left|\Psi_{1}^{\prime}\right\rangle_{S}= & \frac{1}{2}\left(\left|a_{1} b_{1} c_{2}\right\rangle\left|a_{2}^{\prime} b_{2}^{\prime} c_{2}^{\prime}\right\rangle+\left|a_{2} b_{2} c_{2}\right\rangle\left|a_{1}^{\prime} b_{1}^{\prime} c_{2}^{\prime}\right\rangle\right. \\
& \left.+\left|a_{1} b_{2} c_{1}\right\rangle\left|a_{2}^{\prime} b_{1}^{\prime} c_{1}^{\prime}\right\rangle+\left|a_{2} b_{1} c_{1}\right\rangle\left|a_{1}^{\prime} b_{2}^{\prime} c_{1}^{\prime}\right\rangle\right) .
\end{aligned}
$$

If photon pairs $A A^{\prime}$ and $C C^{\prime}$ are in the odd-parity spatial mode and photon pair $B B^{\prime}$ is in the even-parity spatial mode, the spatial-mode DOF of the six-photon system is projected into a mixed state composed of the two pure states $\left|\Psi_{2}^{\prime}\right\rangle_{S}$ and $\left|\Psi_{3}^{\prime}\right\rangle_{S}$. Here

$$
\begin{aligned}
\left|\Psi_{2}^{\prime}\right\rangle_{S}= & \frac{1}{2}\left(\left|a_{1} b_{1} c_{1}\right\rangle\left|a_{2}^{\prime} b_{1}^{\prime} c_{2}^{\prime}\right\rangle+\left|a_{2} b_{2} c_{1}\right\rangle\left|a_{1}^{\prime} b_{2}^{\prime} c_{2}^{\prime}\right\rangle\right. \\
& \left.+\left|a_{1} b_{2} c_{2}\right\rangle\left|a_{2}^{\prime} b_{2}^{\prime} c_{1}^{\prime}\right\rangle+\left|a_{2} b_{1} c_{2}\right\rangle\left|a_{1}^{\prime} b_{1}^{\prime} c_{1}^{\prime}\right\rangle\right), \\
\left|\Psi_{3}^{\prime}\right\rangle_{S}= & \frac{1}{2}\left(\left|a_{1} b_{1} c_{2}\right\rangle\left|a_{2}^{\prime} b_{1}^{\prime} c_{1}^{\prime}\right\rangle+\left|a_{2} b_{2} c_{2}\right\rangle\left|a_{1}^{\prime} b_{2}^{\prime} c_{1}^{\prime}\right\rangle\right. \\
& \left.+\left|a_{1} b_{2} c_{1}\right\rangle\left|a_{2}^{\prime} b_{2}^{\prime} c_{2}^{\prime}\right\rangle+\left|a_{2} b_{1} c_{1}\right\rangle\left|a_{1}^{\prime} b_{1}^{\prime} c_{2}^{\prime}\right\rangle\right)
\end{aligned}
$$

If photon pairs $B B^{\prime}$ and $C C^{\prime}$ are in the odd-parity spatial mode and photon pair $A A^{\prime}$ is in the even-parity spatial mode, the spatial-mode DOF of the six-photon system will project into a mixed state composed of the two pure states $\left|\Psi_{4}^{\prime}\right\rangle_{S}$ and $\left|\Psi_{5}^{\prime}\right\rangle_{S}$. Here

$$
\begin{aligned}
\left|\Psi_{4}^{\prime}\right\rangle_{S}= & \frac{1}{2}\left(\left|a_{1} b_{1} c_{1}\right\rangle\left|a_{1}^{\prime} b_{2}^{\prime} c_{2}^{\prime}\right\rangle+\left|a_{2} b_{2} c_{1}\right\rangle\left|a_{2}^{\prime} b_{1}^{\prime} c_{2}^{\prime}\right\rangle\right. \\
& \left.+\left|a_{1} b_{2} c_{2}\right\rangle\left|a_{1}^{\prime} b_{1}^{\prime} c_{1}^{\prime}\right\rangle+\left|a_{2} b_{1} c_{2}\right\rangle\left|a_{2}^{\prime} b_{2}^{\prime} c_{1}^{\prime}\right\rangle\right), \\
\left|\Psi_{5}^{\prime}\right\rangle_{S}= & \frac{1}{2}\left(\left|a_{1} b_{1} c_{2}\right\rangle\left|a_{1}^{\prime} b_{2}^{\prime} c_{1}^{\prime}\right\rangle+\left|a_{2} b_{2} c_{2}\right\rangle\left|a_{2}^{\prime} b_{1}^{\prime} c_{1}^{\prime}\right\rangle\right. \\
& \left.+\left|a_{1} b_{2} c_{1}\right\rangle\left|a_{1}^{\prime} b_{1}^{\prime} c_{2}^{\prime}\right\rangle+\left|a_{2} b_{1} c_{1}\right\rangle\left|a_{2}^{\prime} b_{2}^{\prime} c_{2}^{\prime}\right\rangle\right) .
\end{aligned}
$$

With the spatial-mode bit-flip operations $\sigma_{x}^{S}$ on the two photons of the three-photon system $A^{\prime} B^{\prime} C^{\prime}$, the spatial-mode states $\left|\Psi_{0}^{\prime}\right\rangle_{S}$ (or $\left|\Psi_{2}^{\prime}\right\rangle_{S},\left|\Psi_{4}^{\prime}\right\rangle_{S}$ ) and $\left|\Psi_{1}^{\prime}\right\rangle_{S}$ (or $\left|\Psi_{3}^{\prime}\right\rangle_{S},\left|\Psi_{5}^{\prime}\right\rangle_{S}$ ) can be transformed into $\left|\Psi_{0}\right\rangle_{S}$ and $\left|\Psi_{1}\right\rangle_{S}$, respectively. After Hadamard operations and detections are performed on the spatial-mode DOF of photons $A^{\prime} B^{\prime} C^{\prime}$ and the conditional phase-flip operation $\sigma_{z}^{S}$ is performed on photon $A$ (or $B, C$ ) [43], states $\left|\varphi^{+}\right\rangle_{S}$ and $\left|\varphi^{-}\right\rangle_{S}$ are obtained with the probabilities $P_{0}^{2}$ and $P_{1}^{2}$, respectively. If the results of the three spatialmode parity-check QNDs have other outcomes, which of the three-photon systems, $A B C$ or $A^{\prime} B^{\prime} C^{\prime}$, has the spatial-mode phase-flip error is ambiguous, which will be discussed in the hyper-EPP with our QSJM in Sec. IVC. States $\left|\varphi^{+}\right\rangle_{S}$ and $\left|\varphi^{-}\right\rangle_{S}$ can be transformed into $\left|\psi_{0}^{+}\right\rangle_{S}$ and $\left|\psi_{0}^{-}\right\rangle_{S}$, respectively, by performing spatialmode Hadamard operations on the three-photon systems.

\section{Hyper-EPP for hyperentangled GHZ states with QSJM}

The hyper-EPP for mixed hyperentangled GHZ states with our QSJM can also be implemented with the quantum circuit shown in Fig. 4. Alice, Bob, and Charlie first perform the same operations [shown in Fig. 廿(a))] on their photon pairs $A A^{\prime}, B B^{\prime}$, and $C C^{\prime}$, respectively. If the results of the P-S-QNDs in a group show that the three photon pairs are all in the even- or odd-parity polarization mode and an odd number of photon pairs are in the even-parity spatial mode, the group of the threephoton systems is preserved.

If the three photon pairs are all in the even- or oddparity polarization mode and an even number of photon pairs are in the even-parity spatial mode in a group, the polarization state of the group can be transferred to the polarization state of another group in which the three photon pairs are not all in the even- or odd-parity polarization mode and an odd number of photon pairs are in the even-parity spatial mode, resorting to the QSJM shown in Fig. 4(b). Then the polarization DOF of the six-photon system $A B C A^{\prime} B^{\prime} C^{\prime}$ is in a mixed state composed of the four pure states $\left|\Psi_{0}\right\rangle_{P},\left|\Psi_{1}\right\rangle_{P},\left|\Psi_{2}\right\rangle_{P}$, and $\left|\Psi_{3}\right\rangle_{P}$, and the spatial-mode DOF of the six-photon system $A B C A^{\prime} B^{\prime} C^{\prime}$ is in a mixed state composed of the 


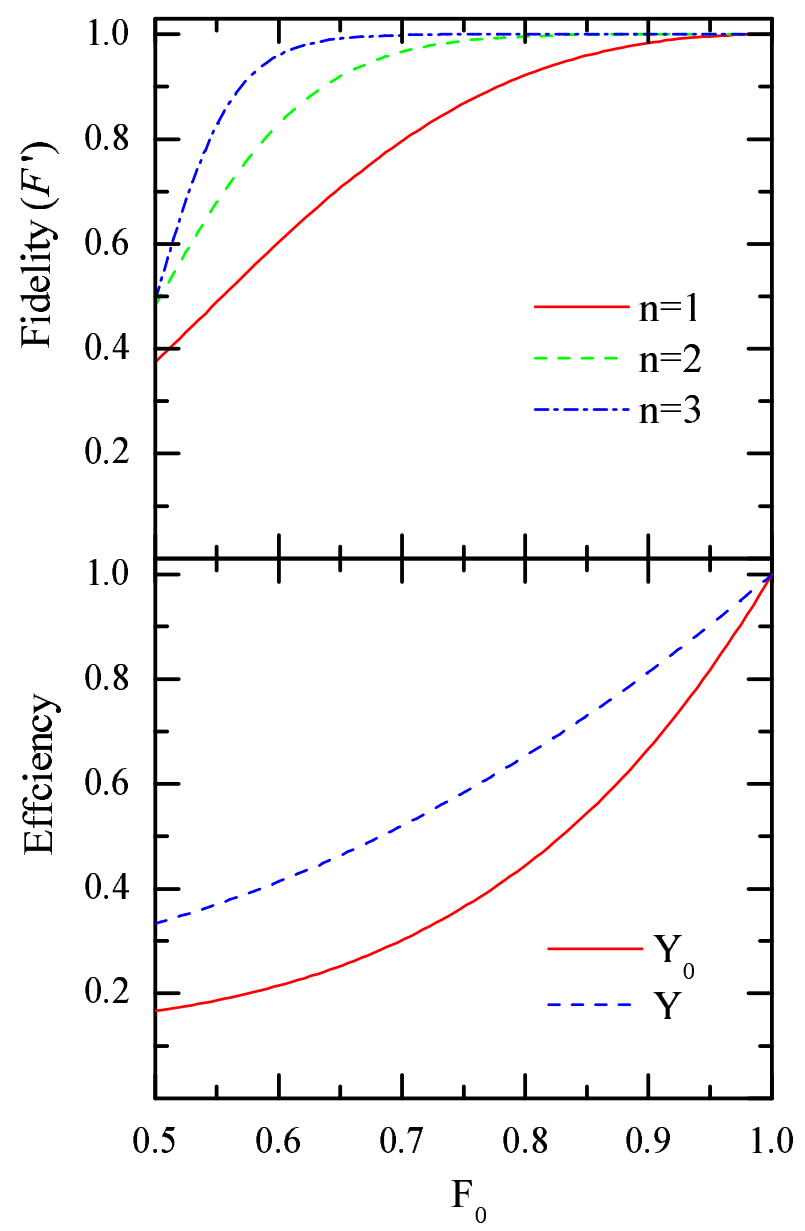

FIG. 6: (Color online) The fidelity and the efficiency of our hyper-EPP for mixed hyperentangled GHZ states. $F^{\prime}=$ $F_{0}^{\prime} P_{0}^{\prime} . \quad n$ is the iteration number of the hyper-EPP process. $Y_{0}$ and $Y$ represent the efficiencies of the first round of the hyper-EPP processes without and with QSJM, respectively. The parameters of the mixed hyperentangled GHZ state are chosen to be $F_{1}=F_{2}=F_{3}$ and $P_{0}=F_{0}$.

two pure states $\left|\Psi_{0}\right\rangle_{S}$ and $\left|\Psi_{1}\right\rangle_{S}$, which is the preserving condition in the first step.

If the three photon pairs are not all in the even- or oddparity polarization mode and an odd number of photon pairs are in the even-parity spatial mode in a group, the spatial-mode state of the group can be transferred to the spatial-mode state of another group in which the three photon pairs are all in the even- or odd-parity polarization mode and an even number of photon pairs are in the even-parity spatial mode, resorting to the QSJM. Then the preserving condition in the first step is achieved. Finally, the group of the three-photon systems has to be discarded if the results of the P-S-QNDs show that the three photon pairs are not all in the even- or odd-parity polarization mode and an even number of photon pairs are in the even-parity spatial mode.

After the first round of this hyper-EPP process with the two steps, the state of three-photon system $A B C$ is transformed into

$$
\begin{aligned}
\rho= & \left(F_{0}^{\prime}\left|\psi_{0}^{+}\right\rangle_{P}\left\langle\psi_{0}^{+}\left|+F_{1}^{\prime}\right| \psi_{1}^{+}\right\rangle_{P}\left\langle\psi_{1}^{+}\right|\right. \\
& \left.+F_{2}^{\prime}\left|\psi_{2}^{+}\right\rangle_{P}\left\langle\psi_{2}^{+}\left|+F_{3}^{\prime}\right| \psi_{3}^{+}\right\rangle_{P}\left\langle\psi_{3}^{+}\right|\right) \\
& \otimes\left(P_{0}^{\prime}\left|\psi_{0}^{+}\right\rangle_{S}\left\langle\psi_{0}^{+}\left|+P_{1}^{\prime}\right| \psi_{0}^{-}\right\rangle_{S}\left\langle\psi_{0}^{-}\right|\right)
\end{aligned}
$$

Here

$$
\begin{aligned}
& F_{0}^{\prime}=\frac{F_{0}^{2}}{F_{0}^{2}+F_{1}^{2}+F_{2}^{2}+F_{3}^{2}}, \\
& F_{1}^{\prime}=\frac{F_{1}^{2}}{F_{0}^{2}+F_{1}^{2}+F_{2}^{2}+F_{3}^{2}}, \\
& F_{2}^{\prime}=\frac{F_{2}^{2}}{F_{0}^{2}+F_{1}^{2}+F_{2}^{2}+F_{3}^{2}}, \\
& F_{3}^{\prime}=\frac{F_{3}^{2}}{F_{0}^{2}+F_{1}^{2}+F_{2}^{2}+F_{3}^{2}}, \\
& P_{0}^{\prime}=\frac{P_{0}^{2}}{P_{0}^{2}+P_{1}^{2}}, \\
& P_{1}^{\prime}=\frac{P_{1}^{2}}{P_{0}^{2}+P_{1}^{2}} .
\end{aligned}
$$

If $P_{0}>1 / 2$, the fidelity $P_{0}^{\prime}>P_{0}$. The fidelity $F_{0}^{\prime}>F_{0}$ if $F_{0}$ satisfies the relation

$$
\begin{aligned}
F_{0}> & \frac{1}{4}\left[3-2 F_{1}-2 F_{2}\right. \\
& \left.-\sqrt{1+4\left(F_{1}+F_{2}\right)-12\left(F_{1}^{2}+F_{2}^{2}\right)-8 F_{1} F_{2}}\right]
\end{aligned}
$$

The fidelity of $\left|\psi_{0}^{+}\right\rangle_{P} \otimes\left|\psi_{0}^{+}\right\rangle_{S}$ in Eq. (29) is $F^{\prime}=F_{0}^{\prime} P_{0}^{\prime}$. With the iteration of our hyper-EPP process, the fidelity of this three-photon state can be improved (shown in Fig. 6 for cases with $F_{1}=F_{2}=F_{3}$ and $P_{0}=F_{0}$ ).

In the hyper-EPP without QSJM, the groups of threephoton systems can only be preserved with the probability of $\left(F_{0}^{2}+F_{1}^{2}+F_{2}^{2}+F_{3}^{2}\right)\left(P_{0}^{2}+P_{1}^{2}\right)$ in the first round of the hyper-EPP process, and the efficiency is

$$
Y_{0}=\left(F_{0}^{2}+F_{1}^{2}+F_{2}^{2}+F_{3}^{2}\right)\left(P_{0}^{2}+P_{1}^{2}\right) .
$$

In this hyper-EPP with our QSJM, the probability of the preserved three-photon systems in the first round of the hyper-EPP process can be increased to

$$
\min \left\{\left(F_{0}^{2}+F_{1}^{2}+F_{2}^{2}+F_{3}^{2}\right),\left(P_{0}^{2}+P_{1}^{2}\right)\right\}
$$

and the efficiency is

$$
Y=\min \left\{\left(P_{0}^{2}+P_{1}^{2}\right),\left(F_{0}^{2}+F_{1}^{2}+F_{2}^{2}+F_{3}^{2}\right)\right\} .
$$

Figure 6 shows the efficiencies of the hyper-EPPs (in the first round) with and without QSJM (for the cases with $F_{1}=F_{2}=F_{3}$ and $P_{0}=F_{0}$ ), and it is obvious that the efficiency of the hyper-EPP with QSJM is greatly improved, compared with the one without QSJM. 


\section{DISCUSSION AND SUMMARY}

The transmission and reflection rule of the doublesided QD-cavity system is the key element for the P-SQND and the QSJM, and it may not be perfect because of decoherence and dephasing. The electron spin decoherence may reduce the fidelity of the proposal, but this effect can be suppressed by extending the electron coherence time to microseconds using spin-echo techniques (using single-photon pluses to play the role of the $\pi$ pulse), which is longer than the cavity photon lifetime $(\sim 10 p \mathrm{~s})$ and the time interval of the input photons (approximately nanoseconds) 62. The exciton dephasing, including optical dephasing and hole spin dephasing, can also reduce the fidelity by a few percent. The hole spin coherence time is three times order of magnitude longer than the cavity photon lifetime [63, 64], and the optical coherence time of the exciton $(\sim 100 p \mathrm{~s})$ is ten times longer than the cavity photon lifetime 65, 66], so the effect of the exciton dephasing may be decreased. The heavy-light hole mixing may also reduce the fidelity by a few percent, but this effect can be decreased by improving the shape, size, and type of QDs [62]. The finestructure splitting could be unaffected by the charged exciton due to the quenched exchange interaction [67, 68]. Also, the Hadamard operation, which is used to transform electron-spin states $|\uparrow\rangle$ and $|\downarrow\rangle$ into $|+\rangle$ and $|-\rangle$, respectively, can be implemented by nanosecond electronspin-resonance microwave pulses or picosecond optical pulses [69].

In the resonant condition $\left(\omega_{c}=\omega_{X^{-}}=\omega_{0}=\omega\right)$, the fidelity of our proposal is mainly reduced by the cavity side leakage and cavity coupling strength (discussed in Appendix (B). The fidelities and the efficiencies of the P-SQND and the QSJM are shown in Figs. 7 and 8 with the coupling strength and cavity side leakage, respectively. Both of these operations work efficiently with a strong coupling strength and a low side leakage and cavity loss rate $\kappa_{s} / \kappa$. The strong coupling strength $g /\left(\kappa+\kappa_{s}\right) \simeq 0.5$ has been observed in a $d=1.5 \mu \mathrm{m}$ micropillar microcavity [70] with a quality factor of $Q \sim 8800$. By improving the sample designs, growth, and fabrication of QDs [1], the coupling strength $g /\left(\kappa+\kappa_{s}\right) \simeq 2.4\left(Q \sim 4 \times 10^{4}\right)$ has been achieved [72] in a $d=1.5 \mu \mathrm{m}$ micropillar microcavity. The fidelities and the efficiencies of of P-S-QND $\left(F_{p}\right.$, $\left.\eta_{p}\right)$ and $\operatorname{QSJM}\left(F_{j}, \eta_{j}\right)$ are $F_{p}=90.4 \%, \eta_{p}=39.6 \%$ and $F_{j}=76.5 \%, \eta_{j}=50 \%$ in the case with $g /\left(\kappa+\kappa_{s}\right) \simeq 0.5$ and $\kappa_{s} / \kappa \simeq 0.3$. They are $F_{p}=92.6 \%, \eta_{p}=60.3 \%$ and $F_{j}=84.4 \%, \eta_{j}=68.5 \%$ for the case with coupling strength $g /\left(\kappa+\kappa_{s}\right) \simeq 2.4$ and $\kappa_{s} / \kappa \simeq 0.3$, and they are $F_{p}=100 \%, \eta_{p}=96.6 \%$ and $F_{j}=99.1 \%, \eta_{j}=97.4 \%$ for the case with side leakage and cavity loss rate $\kappa_{s} / \kappa \simeq 0$ $\left[g /\left(\kappa+\kappa_{s}\right) \simeq 2.4\right]$. The fidelities and the efficiencies of the two operations are mainly reduced by a weak coupling strength and a high cavity side leakage. In experiment, the quality factor of a micropillar microcavity is dominated by the side leakage and cavity loss rate $\kappa_{s} / \kappa$. The side leakage and cavity loss rate $\kappa_{s} / \kappa \simeq 0.7$ has been

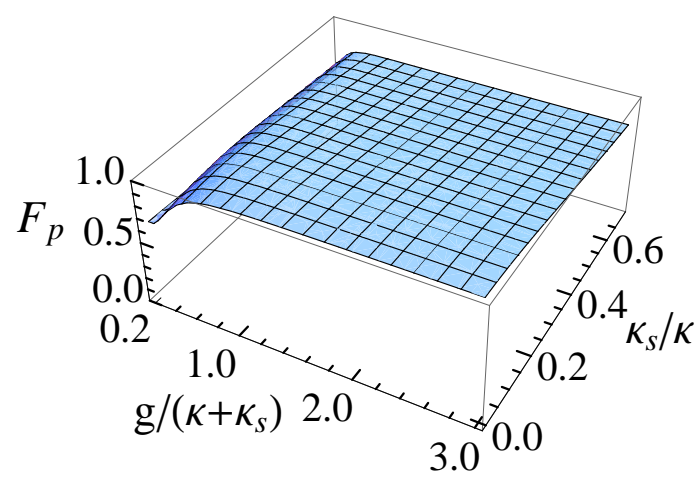

(a)

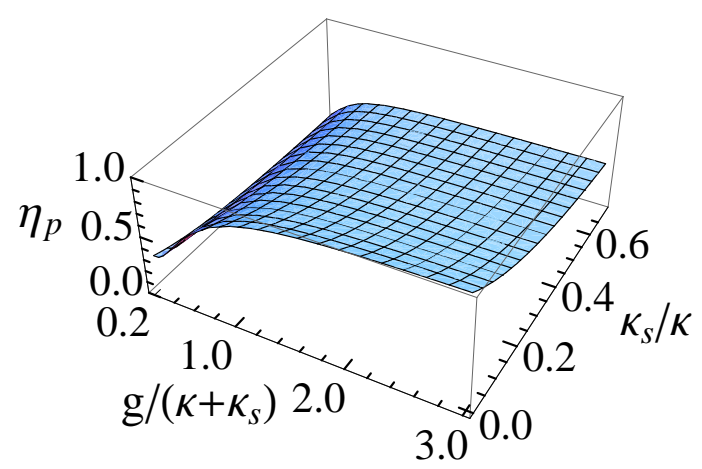

(b)

FIG. 7: (Color online) Fidelity and efficiency of our P-SQND (for the even-parity mode) vs the coupling strength and cavity side leakage rate with $\gamma=0.1 \kappa$.

achieved in a $d=1.5 \mu \mathrm{m}$ micropillar with a quality factor of $Q \sim 1.7 \times 10^{4}\left[\mathrm{~g} /\left(\kappa+\kappa_{s}\right) \simeq 1\right]$ by thinning down the top mirrors [62]. In this case, the fidelities and the efficiencies of the two operations are $F_{p}=78.7 \%, \eta_{p}=39.2 \%$ and $F_{j}=65.9 \%, \eta_{j}=49.6 \%$.

With the P-S-QND and the QSJM, we construct a two-step hyper-EPP for the mixed hyperentangled Bell states with polarization bit-flip errors and spatial-mode phase-flip errors. In the first step, Alice and Bob both perform the P-S-QNDs on their photon pairs. The fidelity and efficiency of this step are $F_{1}=92.7 \%$ and $\eta_{1}=47.6 \%$ in the case with $g /\left(\kappa+\kappa_{s}\right) \simeq 2.4$ and $\kappa_{s} / \kappa \simeq 0.2$, and they are $F_{1}=98 \%$ and $\eta_{2}=65 \%$ in the case with $g /\left(\kappa+\kappa_{s}\right) \simeq 2.4$ and $\kappa_{s} / \kappa \simeq 0.1$ (with P-SQNDs in the even-parity mode). In the second step, Alice and Bob both perform the QSJM on their photon pairs. The fidelity and efficiency of this step are $F_{2}=79.8 \%$ and $\eta_{2}=57.3 \%$ in the case with $g /\left(\kappa+\kappa_{s}\right) \simeq 2.4$ and $\kappa_{s} / \kappa \simeq 0.2$, and they are $F_{2}=88.9 \%$ and $\eta_{2}=72.3 \%$ in the case with $g /\left(\kappa+\kappa_{s}\right) \simeq 2.4$ and $\kappa_{s} / \kappa \simeq 0.1$. [Photons $C$ and $D$ in cases (3) and (4) are detected in the first step to increase the fidelity and the efficiency of the second step, which is identical to detecting photons $C$ and $D$ in the second step.] The efficiency of the hyper- 
EPP is largely reduced by the cavity side leakage. In order to iterate the hyper-EPP process for obtaining highfidelity hyperentangled Bell states, the cavity side leakage should be small in the strong-coupling regime, which means high-efficiency operations in experiment are required to get a stronger coupling strength with a lower side leakage in micropillars.

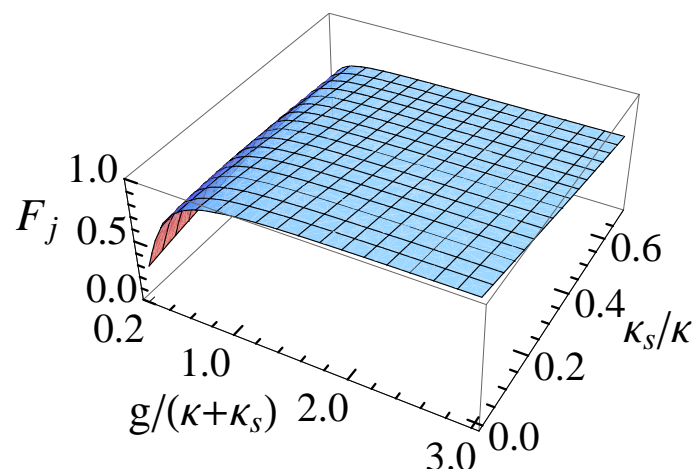

(a)

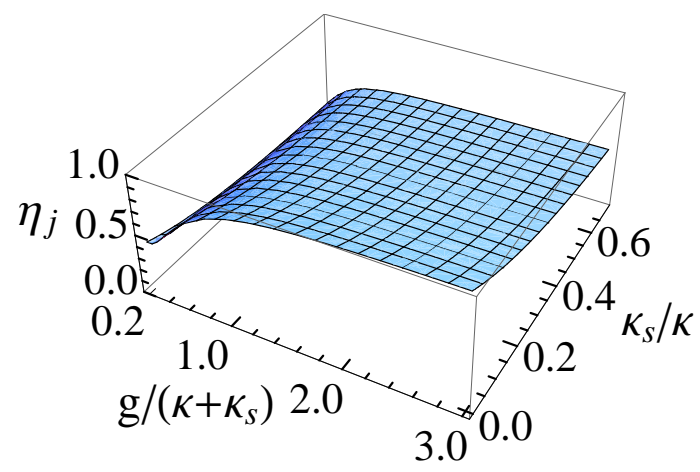

(b)

FIG. 8: (Color online) Fidelity and efficiency of our QSJM (in the present hyper-EPP) vs the coupling strength and cavity side leakage rate with $\gamma=0.1 \kappa$.

In the hyper-EPP without QSJM, the photonic states with only one DOF in the preserving condition have to be discarded. In the present hyper-EPP, the photonic entangled state with only polarization (spatial-mode) DOF in the preserving condition can transfer its polarization (spatial-mode) state to another photonic state with the spatial-mode (polarization) DOF in the preserving condition, which can preserve more high-fidelity entangled photon pairs. That is, the efficiency of a hyper-EPP can be improved by using our QSJM. Moreover, we generalize our hyper-EPP for mixed hyperentangled GHZ states with polarization bit-flip errors and spatial-mode phaseflip errors, which is more complicated than the one for mixed hyperentangled Bell states. This generalization shows that our hyper-EPP with QSJM can also be used to improve the efficiency of the hyper-EPP for mixed multiphoton hyperentangled states.
In summary, we have investigated the possibility of improving the efficiency of the hyper-EPP for mixed hyperentangled states with polarization bit-flip errors and spatial-mode phase-flip errors, resorting to the PS-QND and the QSJM that are constructed with the transmission-reflection rule of double-sided QD-cavity systems. The present two-step hyper-EPP can greatly improve the efficiency by preserving the states that are discarded in the hyper-EPP without QSJM. We have analyzed the experimental feasibility of the two steps of our hyper-EPP, and the analysis shows that they work efficiently in the strong-coupling regime with low cavity side leakage. The present two-step hyper-EPP with our QSJM can be generalized to improve the efficiency of the hyper-EPP for mixed multiphoton hyperentangled states, and it is useful for improving the entanglement of photon systems with several DOFs in long-distance high-capacity quantum communication.

\section{ACKNOWLEDGMENTS}

This work is supported by the National Natural Science Foundation of China under Grants No. 11174039 and No. 11474026 and NECT-11-0031.

\section{Appendix A: QSJM for hyperentangled Bell states}

In this appendix, we discuss the QSJM for hyperentangled Bell states in detail. Let us suppose that the two photon pairs, $A B$ and $A^{\prime} B^{\prime}$, are in different polarization parity modes [Photons $C, D, C^{\prime}, D^{\prime}$ in cases (3) and (4) are detected in the first step, which is identical to detecting photons $C, D, C^{\prime}, D^{\prime}$ in the second step]. That is,

$$
\begin{aligned}
|\phi\rangle_{A B} & =\frac{1}{2}(|R L\rangle+|L R\rangle)_{A B}\left(\left|a_{1} b_{1}\right\rangle+\left|a_{2} b_{2}\right\rangle\right) \\
|\phi\rangle_{A^{\prime} B^{\prime}} & =\frac{1}{2}(|R R\rangle+|L L\rangle)_{A^{\prime} B^{\prime}}\left(\left|a_{1}^{\prime} b_{2}^{\prime}\right\rangle+\left|a_{2}^{\prime} b_{1}^{\prime}\right\rangle\right) .
\end{aligned}
$$

Alice first performs the Hadamard operations on the polarization DOF of photon $A^{\prime}$, and then she puts photon $A^{\prime}$ into the quantum circuit shown in Fig. 2(a). After these operations are performed on photon $A^{\prime}$, Bob performs the same operations as those performed by Alice on photon $B^{\prime}$. After photon $B^{\prime}$ passes through the quantum circuit, Alice and Bob perform the Hadamard operations on electron spins $e_{a}$ and $e_{b}$, and the state of the quantum system composed of QDs and photons $A^{\prime}$ and $B^{\prime}$ is transformed from $\left|\Phi_{0}\right\rangle_{A^{\prime} B^{\prime} e_{a} e_{b}}$ to $\left|\Phi_{1}\right\rangle_{A^{\prime} B^{\prime} e_{a} e_{b}}$. Here

$$
\begin{aligned}
\left|\Phi_{0}\right\rangle_{A^{\prime} B^{\prime} e_{a} e_{b}}= & \frac{1}{2}(|\uparrow\rangle+|\downarrow\rangle)_{e_{a}} \otimes(|\uparrow\rangle+|\downarrow\rangle)_{e_{b}} \\
& \otimes|\phi\rangle_{A^{\prime} B^{\prime}} \\
\left|\Phi_{1}\right\rangle_{A^{\prime} B^{\prime} e_{a} e_{b}}= & \frac{1}{4}\left[(|R R\rangle+|L L\rangle)_{A^{\prime} B^{\prime}}(|\uparrow \uparrow\rangle+|\downarrow \downarrow\rangle)_{e_{a} e_{b}}\right.
\end{aligned}
$$




$$
\begin{aligned}
& \left.+(|R L\rangle+|L R\rangle)_{A^{\prime} B^{\prime}}(|\uparrow \uparrow\rangle-|\downarrow \downarrow\rangle)_{e_{a} e_{b}}\right] \\
& \otimes\left(\left|a_{1}^{\prime} b_{2}^{\prime}\right\rangle+\left|a_{2}^{\prime} b_{1}^{\prime}\right\rangle\right) .
\end{aligned}
$$

The polarization states of photons $A^{\prime}$ and $B^{\prime}$ are measured in the orthogonal basis $\{|R\rangle,|L\rangle\}$. If the two photons are in the even-parity polarization mode, the polarization state of the two-photon system $A^{\prime} B^{\prime}$ is transferred to the state of electron spins $e_{a}$ and $e_{b}$. If the polarization state of the photon pair $A^{\prime} B^{\prime}$ is in the odd-parity polarization mode, a phase-flip operation $\sigma_{z}=|\uparrow\rangle\langle\uparrow|-| \downarrow\rangle\langle\downarrow|$ must be performed on electron spin $e_{a}$.

Subsequently, photon $A$ is put into the quantum circuit shown in Fig. 2 (a) by Alice. After photon $A$ passes through the quantum circuit, Alice performs the Hadamard operations on the polarization DOF of photon $A$ and electron spin $e_{a}$. Then photon $A$ is put into the quantum circuit again. After these operations are performed on photon $A$, Bob performs the same operations as those performed by Alice on photon $B$ and electron spin $e_{b}$. Finally, Alice and Bob perform the Hadamard operations on electron spins $e_{a}$ and $e_{b}$, and the state of the quantum system composed of QDs and photons $A$ and $B$ are transformed from $\left|\Phi_{1}\right\rangle_{A B e_{a} e_{b}}$ to $\left|\Phi_{2}\right\rangle_{A B e_{a} e_{b}}$. Here

$$
\begin{aligned}
\left|\Phi_{1}\right\rangle_{A B e_{a} e_{b}}= & \frac{1}{\sqrt{2}}(|\uparrow \uparrow\rangle+|\downarrow \downarrow\rangle) e_{a} e_{b} \otimes|\phi\rangle_{A B}, \\
\left|\Phi_{2}\right\rangle_{A B e_{a} e_{b}}= & \frac{1}{2 \sqrt{2}}(|R R\rangle-|L L\rangle)_{A B}(|\uparrow \downarrow\rangle+|\downarrow \uparrow\rangle)_{e_{a} e_{b}} \\
& \otimes\left(\left|a_{1} b_{1}\right\rangle+\left|a_{2} b_{2}\right\rangle\right) .
\end{aligned}
$$

Electron spins $e_{a}$ and $e_{b}$ are measured in the orthogonal basis $\{|\uparrow\rangle,|\downarrow\rangle\}$. If the two electron spins are in the odd-parity mode, a phase-flip operation $\sigma_{z}^{P}$ performed on photon $A$ is required to transfer the state of electron spins $e_{a}$ and $e_{b}$ to the polarization state of photon pair $A B$.

If the two photon pairs $A B$ and $A^{\prime} B^{\prime}$ are in the same polarization parity mode,

$$
\begin{aligned}
\left|\phi^{\prime}\right\rangle_{A B} & =\frac{1}{2}(|R R\rangle+|L L\rangle)_{A B}\left(\left|a_{1} b_{1}\right\rangle+\left|a_{2} b_{2}\right\rangle\right), \\
\left|\phi^{\prime}\right\rangle_{A^{\prime} B^{\prime}} & =\frac{1}{2}(|R R\rangle+|L L\rangle)_{A^{\prime} B^{\prime}}\left(\left|a_{1}^{\prime} b_{2}^{\prime}\right\rangle+\left|a_{2}^{\prime} b_{1}^{\prime}\right\rangle\right),
\end{aligned}
$$

Alice and Bob can perform the same QSJM operation on the two photon pairs. The finial state $\left|\Phi_{2}^{\prime}\right\rangle_{A B e_{a} e_{b}}$ will become

$$
\begin{aligned}
\left|\Phi_{2}^{\prime}\right\rangle_{A B e_{a} e_{b}}= & \frac{1}{2 \sqrt{2}}(|R R\rangle+|L L\rangle)_{A B}(|\uparrow \uparrow\rangle+|\downarrow \downarrow\rangle)_{e_{a} e_{b}} \\
& \otimes\left(\left|a_{1} b_{1}\right\rangle+\left|a_{2} b_{2}\right\rangle\right) .
\end{aligned}
$$

The two electron spins are in the even-parity mode, and the polarization state of the photon pair $A^{\prime} B^{\prime}$ is transferred to the polarization state of the photon pair $A B$.

If the two photon pairs $A B$ and $A^{\prime} B^{\prime}$ are in the different polarization parity modes,

$$
\left|\phi^{\prime}\right\rangle_{A B}=\frac{1}{2}(|R R\rangle+|L L\rangle)_{A B}\left(\left|a_{1} b_{1}\right\rangle+\left|a_{2} b_{2}\right\rangle\right)
$$

$$
\left|\phi^{\prime \prime}\right\rangle_{A^{\prime} B^{\prime}}=\frac{1}{2}(|R L\rangle+|L R\rangle)_{A^{\prime} B^{\prime}}\left(\left|a_{1}^{\prime} b_{2}^{\prime}\right\rangle+\left|a_{2}^{\prime} b_{1}^{\prime}\right\rangle\right)
$$

Alice and Bob can also perform the same QSJM operation on the two photon pairs. The finial state $\left|\Phi_{2}^{\prime \prime}\right\rangle_{A B e_{a} e_{b}}$ will become

$$
\begin{aligned}
\left|\Phi_{2}^{\prime \prime}\right\rangle_{A B e_{a} e_{b}}= & \frac{1}{2 \sqrt{2}}(|R R\rangle-|L L\rangle)_{A B}(|\uparrow \uparrow\rangle+|\downarrow \downarrow\rangle)_{e_{a} e_{b}} \\
& \otimes\left(\left|a_{1} b_{1}\right\rangle+\left|a_{2} b_{2}\right\rangle\right) .
\end{aligned}
$$

The two electron spins are in the even-parity mode, and the polarization state of the photon pair $A^{\prime} B^{\prime}$ is transferred to the polarization state of the photon pair $A B$ after the Hadamard operations are performed on the polarization DOF of the photon pair $A B$.

If both photon pairs $A B$ and $A^{\prime} B^{\prime}$ are in the same polarization parity mode,

$$
\begin{aligned}
\left|\phi^{\prime \prime}\right\rangle_{A B} & =\frac{1}{2}(|R L\rangle+|L R\rangle)_{A B}\left(\left|a_{1} b_{1}\right\rangle+\left|a_{2} b_{2}\right\rangle\right), \\
\left|\phi^{\prime \prime \prime}\right\rangle_{A^{\prime} B^{\prime}} & =\frac{1}{2}(|R L\rangle+|L R\rangle)_{A^{\prime} B^{\prime}}\left(\left|a_{1}^{\prime} b_{2}^{\prime}\right\rangle+\left|a_{2}^{\prime} b_{1}^{\prime}\right\rangle\right),
\end{aligned}
$$

Alice and Bob can perform the same QSJM operation on the two photon pairs as well. The finial state $\left|\Phi_{2}^{\prime \prime \prime}\right\rangle_{A B e_{a} e_{b}}$ will become

$$
\begin{aligned}
\left|\Phi_{2}^{\prime \prime \prime}\right\rangle_{A B e_{a} e_{b}}= & \frac{1}{2 \sqrt{2}}(|R R\rangle+|L L\rangle)_{A B}(|\uparrow \downarrow\rangle+|\downarrow \uparrow\rangle)_{e_{a} e_{b}} \\
& \otimes\left(\left|a_{1} b_{1}\right\rangle+\left|a_{2} b_{2}\right\rangle\right) .
\end{aligned}
$$

The two electron spins are in the odd-parity mode, and the polarization state of photon pair $A^{\prime} B^{\prime}$ is transferred to the polarization state of photon pair $A B$ after a phaseflip operation $\sigma_{z}^{P}$ is performed on photon $A$ and the Hadamard operations are performed on the polarization DOF of photon pair $A B$.

\section{Appendix B: Fidelities and efficiencies of P-S-QND and QSJM}

In the resonant condition $\left(\omega_{c}=\omega_{X^{-}}=\omega_{0}=\omega\right)$, the fidelity of our proposal is mainly reduced by the cavity side leakage and cavity coupling strength. The transmission and reflection rule in Eq. (4) is reduced to

$$
\begin{aligned}
& \left|R^{\uparrow}, i_{2}, \uparrow\right\rangle \rightarrow|r|\left|L^{\downarrow}, i_{2}, \uparrow\right\rangle-|t|\left|R^{\uparrow}, i_{1}, \uparrow\right\rangle, \\
& \left|L^{\downarrow}, i_{1}, \uparrow\right\rangle \rightarrow|r|\left|R^{\uparrow}, i_{1}, \uparrow\right\rangle-|t|\left|L^{\downarrow}, i_{2}, \uparrow\right\rangle, \\
& \left|R^{\downarrow}, i_{1}, \downarrow\right\rangle \rightarrow|r|\left|L^{\uparrow}, i_{1}, \downarrow\right\rangle-|t|\left|R^{\downarrow}, i_{2}, \downarrow\right\rangle, \\
& \left|L^{\uparrow}, i_{2}, \downarrow\right\rangle \rightarrow|r|\left|R^{\downarrow}, i_{2}, \downarrow\right\rangle-|t|\left|L^{\uparrow}, i_{1}, \downarrow\right\rangle, \\
& \left|R^{\downarrow}, i_{1}, \uparrow\right\rangle \rightarrow-\left|t_{0}\right|\left|R^{\downarrow}, i_{2}, \uparrow\right\rangle+\left|r_{0}\right|\left|L^{\uparrow}, i_{1}, \uparrow\right\rangle, \\
& \left|L^{\uparrow}, i_{2}, \uparrow\right\rangle \rightarrow-\left|t_{0}\right|\left|L^{\uparrow}, i_{1}, \uparrow\right\rangle+\left|r_{0}\right|\left|R^{\downarrow}, i_{2}, \uparrow\right\rangle, \\
& \left|R^{\uparrow}, i_{2}, \downarrow\right\rangle \rightarrow-\left|t_{0}\right|\left|R^{\uparrow}, i_{1}, \downarrow\right\rangle+\left|r_{0}\right|\left|L^{\downarrow}, i_{2}, \downarrow\right\rangle, \\
& \left|L^{\downarrow}, i_{1}, \downarrow\right\rangle \rightarrow-\left|t_{0}\right|\left|L^{\downarrow}, i_{2}, \downarrow\right\rangle+\left|r_{0}\right|\left|R^{\uparrow}, i_{1}, \downarrow\right\rangle .
\end{aligned}
$$


The fidelity of a quantum information process is defined as $F=\left|\left\langle\psi_{f} \mid \psi\right\rangle\right|^{2}$, where $|\psi\rangle$ is the ideal final state of the system after the quantum information processing and $\left|\psi_{f}\right\rangle$ is the final state of the system found by considering an experimental environment. The efficiency of a photonic quantum information process is defined as the probability of the photons to being detected after quantum information processing in a practical experimental environment. The fidelity and the efficiency of our P-SQND (for the even-parity mode) can be described as

$$
\begin{aligned}
& F_{p}=\frac{\left(\sum_{i=1}^{4} m_{i}\right)^{2}}{4\left(\sum_{j=1}^{4} m_{j}^{2}+\sum_{k=5}^{9} m_{k}\right)}, \\
& \eta_{p}=\frac{1}{16}\left(|r|^{2}+|t|^{2}+\left|t_{0}\right|^{2}+\left|r_{0}\right|^{2}\right)^{4} .
\end{aligned}
$$

The fidelity and the efficiency of our QSJM (in the present hyper-EPP process) can be described as

$$
\begin{aligned}
& F_{j}=\frac{\left(|r|+|t|+\left|r_{0}\right|+\left|t_{0}\right|\right)^{2}\left(|r|+\left|t_{0}\right|\right)^{4}}{2 \sum_{k=1}^{8} n_{k}^{2}\left[(|r|+|t|)^{2}+\left(\left|r_{0}\right|+\left|t_{0}\right|\right)^{2}\right]} \\
& \eta_{j}=\frac{1}{8}\left(|r|^{2}+|t|^{2}+\left|t_{0}\right|^{2}+\left|r_{0}\right|^{2}\right)^{3} .
\end{aligned}
$$

Here

$m_{1}=\left(|t|^{2}+|r|^{2}\right)\left|t_{0}\right|^{2}+\left(\left|t_{0}\right|^{2}+\left|r_{0}\right|^{2}\right)\left|r_{0}\right|^{2}$

$$
\begin{aligned}
& m_{2}=\left(|t|^{2}+|r|^{2}\right)|r|^{2}+\left(\left|t_{0}\right|^{2}+\left|r_{0}\right|^{2}\right)|t|^{2}, \\
& m_{3}=\left(|t|^{2}+|r|^{2}\right)\left|r_{0}\right|^{2}+\left(\left|t_{0}\right|^{2}+\left|r_{0}\right|^{2}\right)\left|t_{0}\right|^{2} \text {, } \\
& m_{4}=\left(|t|^{2}+|r|^{2}\right)|t|^{2}+\left(\left|t_{0}\right|^{2}+\left|r_{0}\right|^{2}\right)|r|^{2} \text {, } \\
& m_{5}=2\left(\left|t_{0}\right|^{2}\left|r_{0}\right|^{2}+|r|^{2}|t|^{2}\right)\left(\left|t_{0}\right|^{2}+|t|^{2}+\left|r_{0}\right|^{2}+|r|^{2}\right)^{2} \text {, } \\
& m_{6}=4\left|t_{0}\right|^{2}|r|^{2}\left(|t|^{2}+\left|r_{0}\right|^{2}\right)^{2} \text {, } \\
& m_{7}=4\left|t_{0}\right|^{2}|t|^{2}\left(\left|r_{0}\right|^{2}+|r|^{2}\right)^{2} \text {, } \\
& m_{8}=4\left|r_{0}\right|^{2}|r|^{2}\left(\left|t_{0}\right|^{2}+|t|^{2}\right)^{2} \text {, } \\
& m_{9}=4\left|r_{0}\right|^{2}|t|^{2}\left(\left|t_{0}\right|^{2}+|r|^{2}\right)^{2} \text {, } \\
& n_{1}=(|t|+|r|)|r|+(|r|-|t|)|t|+\left(\left|t_{0}\right|+\left|r_{0}\right|\right)|r| \\
& -\left(\left|t_{0}\right|-\left|r_{0}\right|\right)|t| \\
& n_{2}=(|t|+|r|)|t|+(|r|-|t|)|r|+\left(\left|t_{0}\right|+\left|r_{0}\right|\right)|t| \\
& -\left(\left|t_{0}\right|-\left|r_{0}\right|\right)|r| \text {, } \\
& n_{3}=\left(\left|t_{0}\right|+\left|r_{0}\right|\right)\left|t_{0}\right|-\left(\left|r_{0}\right|-\left|t_{0}\right|\right)\left|r_{0}\right|+|(|t|+|r|)| t_{0} \mid \\
& -(|r|-|t|)\left|r_{0}\right| \\
& n_{4}=\left(\left|t_{0}\right|+\left|r_{0}\right|\right)\left|r_{0}\right|-\left(\left|r_{0}\right|-\left|t_{0}\right|\right)\left|t_{0}\right|+|(|t|+|r|)| r_{0} \mid \\
& -(|r|-|t|)\left|t_{0}\right| \\
& n_{5}=(|t|+|r|)\left|t_{0}\right|+(|r|-|t|)\left|r_{0}\right|+\left(\left|t_{0}\right|+\left|r_{0}\right|\right)\left|t_{0}\right| \\
& -\left(\left|t_{0}\right|+\left|r_{0}\right|\right)\left|r_{0}\right| \text {, } \\
& n_{6}=(|t|+|r|)\left|r_{0}\right|+(|r|-|t|)\left|t_{0}\right|+\left(\left|t_{0}\right|+\left|r_{0}\right|\right)\left|r_{0}\right| \\
& -\left(\left|t_{0}\right|+\left|r_{0}\right|\right)\left|t_{0}\right| \text {, } \\
& n_{7}=\left(\left|t_{0}\right|+\left|r_{0}\right|\right)|r|-\left(\left|r_{0}\right|-\left|t_{0}\right|\right)|t|+|(|t|+|r|)| r \mid \\
& -(|r|-|t|)|t| \\
& n_{8}=\left(\left|t_{0}\right|+\left|r_{0}\right|\right)|t|-\left(\left|r_{0}\right|-\left|t_{0}\right|\right)|r|+|(|t|+|r|)| t \mid \\
& -(|r|-|t|)|r| \text {. }
\end{aligned}
$$

[1] M. A. Nielsen and I. L. Chuang, Quantum Computation and Quantum Information (Cambridge University Press, Cambridge, 2000).

[2] C. H. Bennett, G. Brassard, C. Crépeau, R. Jozsa, A. Peres, and W. K. Wootters, Phys. Rev. Lett. 70, 1895 (1993).

[3] C. H. Bennett and S. J. Wiesner, Phys. Rev. Lett. 69, 2881 (1992).

[4] X. S. Liu, G. L. Long, D. M. Tong, and F. Li, Phys. Rev. A 65, 022304 (2002)

[5] N. Gisin, G. Ribordy, W. Tittel, and H. Zbinden, Rev. Mod. Phys. 74, 145 (2002).

[6] A. K. Ekert, Phys. Rev. Lett. 67, 661 (1991).

[7] C. H. Bennett, G. Brassard, and N. D. Mermin, Phys. Rev. Lett. 68, 557 (1992).

[8] X. H. Li, F. G. Deng, and H. Y. Zhou, Phys. Rev. A 78, 022321 (2008).

[9] G. L. Long and X. S. Liu, Phys. Rev. A 65, 032302 (2002).

[10] F. G. Deng, G. L. Long, and X. S. Liu, Phys. Rev. A 68, 042317 (2003).

[11] M. Hillery, V. Bužek, and A. Berthiaume, Phys. Rev. A 59, 1829 (1999).

[12] A. Karlsson, M. Koashi, and N. Imoto, Phys. Rev. A 59, 162 (1999).
[13] L. Xiao, G. L. Long, F. G. Deng, and J. W. Pan, Phys. Rev. A 69, 052307 (2004).

[14] J. T. Barreiro, N. K. Langford, N. A. Peters, and P. G. Kwiat, Phys. Rev. Lett. 95, 260501 (2005).

[15] W. B. Gao, C. Y. Lu, X. C. Yao, P. Xu, O. Gühne, A. Goebel, Y. A. Chen, C. Z. Peng, Z. B. Chen, and J. W. Pan, Nat. Phys. 6, 331 (2010).

[16] G. Vallone, R. Ceccarelli, F. De Martini, and P. Mataloni, Phys. Rev. A 79, 030301(R) (2009).

[17] B. C. Ren, H. R. Wei, and F. G. Deng, Laser phys. Lett. 10, 095202 (2013).

[18] B. C. Ren and F. G. Deng, Sci. Rep. 4, 4623 (2014).

[19] J. T. Barreiro, T. C. Wei, and P. G. Kwiat, Nat. Phys. 4, 282 (2008).

[20] Y. B. Sheng, F. G. Deng, and G. L. Long, Phys. Rev. A 82, 032318 (2010).

[21] B. C. Ren, H. R. Wei, M. Hua, T. Li, and F. G. Deng, Opt. Express 20, 24664 (2012).

[22] T. C. Wei, J. T. Barreiro, and P. G. Kwiat, Phys. Rev. A 75, 060305(R) (2007).

[23] N. Pisenti, C. P. E. Gaebler, and T. W. Lynn, Phys. Rev. A 84, 022340 (2011).

[24] T. J. Wang, Y. Lu, and G. L. Long, Phys. Rev. A 86, 042337 (2012).

[25] B. C. Ren, F. F. Du, and F. G. Deng, Phys. Rev. A 88, 
$012302(2013)$.

[26] B. C. Ren and G. L. Long, Opt. Express 22, 6547 (2014).

[27] Z. B. Chen, J. W. Pan, Y. D. Zhang, Č Brukner, and A. Zeilinger, Phys. Rev. Lett. 90, 160408 (2003).

[28] T. Yang, Q. Zhang, J. Zhang, J. Yin, Z. Zhao, M. Żukowski, Z. B. Chen, and J. W. Pan, Phys. Rev. Lett. 95, 240406 (2005).

[29] T. J. Wang, S. Y. Song, and G. L. Long, Phys. Rev. A 85, 062311 (2012).

[30] P. G. Kwiat and H. Weinfurter, Phys. Rev. A 58, R2623 (1998).

[31] S. P. Walborn, S. Pádua, and C. H. Monken, Phys. Rev. A 68, 042313 (2003).

[32] C. Schuck, G. Huber, C. Kurtsiefer, and H. Weinfurter, Phys. Rev. Lett. 96, 190501 (2006).

[33] M. Barbieri, G. Vallone, P. Mataloni, and F. De Martini, Phys. Rev. A 75, 042317 (2007).

[34] Y. B. Sheng and F. G. Deng, Phys. Rev. A 81, 032307 (2010).

[35] Y. B. Sheng and F. G. Deng, Phys. Rev. A 82, 044305 (2010).

[36] X. H. Li, Phys. Rev. A 82, 044304 (2010).

[37] F. G. Deng, Phys. Rev. A 83, 062316 (2011).

[38] Y. B. Sheng and L. Zhou, Laser Phys. Lett. 11, 085203 (2014).

[39] C. H. Bennett, G. Brassard, S. Popescu, B. Schumacher, J. A. Smolin, and W. K. Wootters, Phys. Rev. Lett. 76, 722 (1996).

[40] D. Deutsch, A. Ekert, R. Jozsa, C. Macchiavello, S. Popescu, and A. Sanpera, Phys. Rev. Lett. 77, 2818 (1996).

[41] Y. B. Sheng, F. G. Deng, and H. Y. Zhou, Phys. Rev. A 77, 042308 (2008).

[42] M. Murao, M. B. Plenio, S. Popescu, V. Vedral, and P. L. Knight, Phys. Rev. A 57, R4075 (1998).

[43] F. G. Deng, Phys. Rev. A 84, 052312 (2011).

[44] C. Wang, Y. Zhang, and R. Zhang, Opt. Express 19, 25685 (2011).

[45] C. Wang, Y. Zhang, and G. S. Jin, Phys. Rev. A 84, 032307 (2011).

[46] B. C. Ren and F. G. Deng, Laser phys. Lett. 10, 115201 (2013).

[47] C. Cao, C. Wang, L. Y. He, and R. Zhang, Opt. Express 21, 4093 (2013).

[48] Y. B. Sheng, L. Zhou, and G. L. Long, Phys. Rev. A 88, 022302 (2013).

[49] J. W. Pan, C. Simon, Č. Brukner, and A. Zeilinger, Nature (London) 410, 1067 (2001).

[50] C. Simon and J. W. Pan, Phys. Rev. Lett. 89, 257901 (2002).

[51] J. W. Pan, S. Gasparoni, R. Ursin, G. Weihs, and A. Zeilinger, Nature (London) 423, 417 (2003).

[52] M. Czechlewski, A. Grudka, S. Ishizaka, and A. Wójcik, Phys. Rev. A 80, 014303 (2009).

[53] D. Gonta and P. van Loock, Phys. Rev. A 86, 052312
(2012).

[54] K. Maruyama and F. Nori, Phys. Rev. A 78, 022312 (2008).

[55] C. Vitelli, N. Spagnolo, L. Aparo, F. Sciarrino, E. Santamato, and L. Marrucci, Nat. photon. 7, 521 (2013).

[56] J. Gudat, C. Bonato, E. van Nieuwenburg, S. Thon, H Kim, P. M. Petroff, M. P. van Exter, and D. Bouwmeester, Appl. Phys. Lett. 98, 121111 (2011).

[57] R. J. Warburton, C. S. Dürr, K. Karrai, J. P. Kotthaus, G. Medeiros-Ribeiro, and P. M. Petroff, Phys. Rev. Lett. 79, 5282 (1997).

[58] C. Y. Hu, W. Ossau, D. R. Yakovlev, G. Landwehr, T. Wojtowicz, G. Karczewski, and J. Kossut, Phys. Rev. B 58, R1766 (1998).

[59] D. F. Walls and G. J. Milburn, Quantum Optics (Springer-Verlag, Berlin, 1994).

[60] C. Y. Hu, W. J. Munro, J. L. O'Brien, and J. G. Rarity, Phys. Rev. B 80, 205326 (2009).

[61] X. H. Li, F. G. Deng and H. Y. Zhou, Appl. Phys. Lett. 91, 144101 (2007).

[62] C. Y. Hu and J. G. Rarity, Phys. Rev. B 83, 115303 (2011).

[63] B. D. Gerardot, D. Brunner, P. A. Dalgarno, P. Öhberg, S. Seidl, M. Kroner, K. Karrai, N. G. Stoltz, P. M. Petroff, and R. J. Warburton, Nature (London) 451, 441 (2008).

[64] D. Brunner, B. D. Gerardot, P. A. Dalgarno, G. Wüst, K. Karrai, N. G. Stoltz, P. M. Petroff, and R. J. Warburton, Science 325, 70 (2009).

[65] P. Borri, W. Langbein, S. Schneider, U. Woggon, R. L. Sellin, D. Ouyang, and D. Bimberg, Phys. Rev. Lett. 87, 157401 (2001).

[66] D. Birkedal, K. Leosson, and J. M. Hvam, Phys. Rev. Lett. 87, 227401 (2001).

[67] M. Bayer, G. Ortner, O. Stern, A. Kuther, A. A. Gorbunov, A. Forchel, P. Hawrylak, S. Fafard, K. Hinzer, T. L. Reinecke, S. N. Walck, J. P. Reithmaier, F. Klopf, and F. Schäfer, Phys. Rev. B 65, 195315 (2002).

[68] J. J. Finley, D. J. Mowbray, M. S. Skolnick, A. D. Ashmore, C. Baker, A. F. G. Monte, and M. Hopkinson, Phys. Rev. B 66, 153316 (2002).

[69] D. Press, T. D. Ladd, B. Zhang, and Y. Yamamoto, Nature (London) 456, 218 (2008).

[70] J. P. Reithmaier, G. Sȩk, A. Löffler, C. Hofmann, S. Kuhn, S. Reitzenstein, L. V. Keldysh, V. D. Kulakovskii, T. L. Reinecke, and A. Forchel, Nature (London) 432, 197 (2004).

[71] S. Reitzenstein, C. Hofmann, A. Gorbunov, M. Strauß, S. H. Kwon, C. Schneider, A. Löffler, S. Höfling, M. Kamp, and A. Forchel, Appl. Phys. Lett. 90, 251109 (2007).

[72] T. Yoshie, A. Scherer, J. Hendrickson, G. Khitrova, H. M. Gibbs, G. Rupper, C. Ell, O. B. Shchekin, and D. G. Deppe, Nature (London) 432, 200 (2004). 